\title{
Sulfation of $\mathbf{O}$-glycans on Mucin-type Proteins From Serous Ovarian Epithelial Tumors
}

\section{Authors}

Kristina A. Thomsson, Varvara Vitiazeva, Constantina Mateoiu, Chunsheng Jin, Jining Liu, Jan Holgersson, Birgitta Weijdegård, Karin Sundfeldt, and Niclas G. Karlsson

Correspondence

niclaska@oslomet.no

\section{In Brief}

Ovarian cancer cells secrete $\mathrm{O}-$ glycosylated sulfated proteins, probably with a purpose to confuse the immune system. We show that in addition to the ubiquitous sulfation on $\mathrm{N}$ acetylglucosamine, we detected the less prevalent sulfation reported on galactose. Selecting three sulfotransferases that are capable of galactose sulfation, the ovarian sulfation could be mimicked recombinantly. One of these transferases (Gal3ST2) was shown to be downregulated in malignant cancer compared to benign. The data highlights that sulfation is involved in ovarian cancer development.

\section{Graphical Abstract}

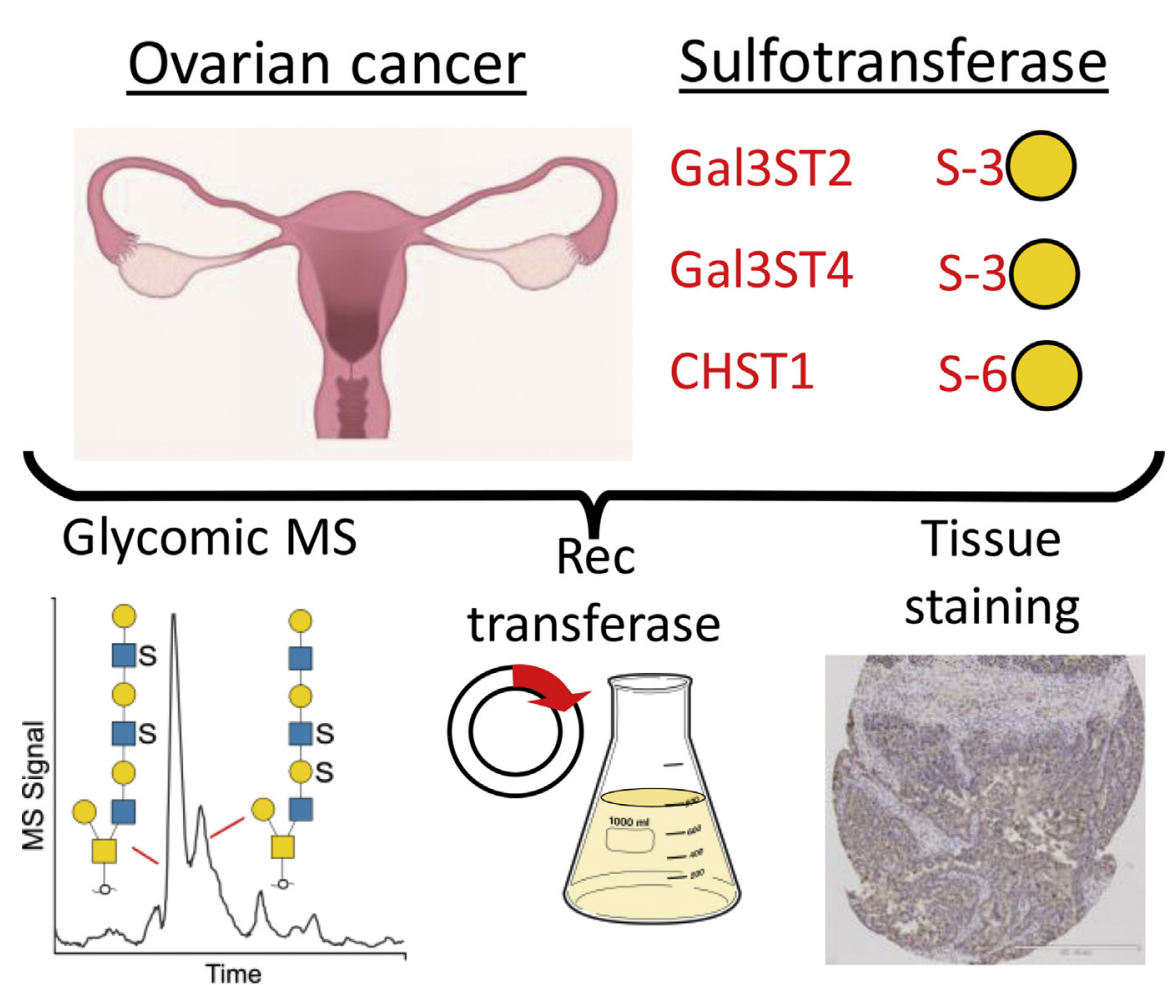

\section{Highlights}

- Ovarian cancer tissue contains highly sulfated O-glycoproteins.

- Sulfation occurs on GlcNAc (6-position) and Gal (3- and 6-position).

- Sulfation of Gal can be mimicked recombinantly with selected sulfotransferase.

- The Gal3ST2 sulfotransferase level is lower in malignant cancer compared with benign. 


\title{
Sulfation of $\mathrm{O}$-glycans on Mucin-type Proteins From Serous Ovarian Epithelial Tumors
}

\author{
Kristina A. Thomsson ${ }^{1, \ddagger}$, Varvara Vitiazeva ${ }^{1, \neq}$, Constantina Mateoiu ${ }^{2}$, Chunsheng Jin ${ }^{1}$, \\ Jining Liu $^{3}$, Jan Holgersson ${ }^{3} \odot$, Birgitta Weijdegård ${ }^{4}$, Karin Sundfeldt ${ }^{4, \S_{\odot}}$, and \\ Niclas G. Karlsson ${ }^{1,5,}{ }^{, \S}$
}

Despite sulfated $\boldsymbol{O}$-linked glycans being abundant on ovarian cancer $(\mathrm{OC})$ glycoproteins, their regulation during cancer development and involvement in cancer pathogenesis remain unexplored. We characterized $\mathbf{O}$-glycans carrying sulfation on galactose residues and compared their expression with defined sulfotransferases regulated during OC development. Desialylated sulfated oligosaccharides were released from acidic glycoproteins in the cyst fluid from one patient with a benign serous cyst and one patient with serous OC. Oligosaccharides characterized by LC-MS ${ }^{n}$ were identified as core 1 and core 2 0 -glycans up to the size of decamers and with 1 to 4 sulfates linked to GICNAc residues and to C-3 and/or C-6 of Gal. To study the specificity of the potential ovarian sulfotransferases involved, Gal3ST2 (Gal-3S)-, Gal3ST4 (Gal-3S)-, and CHST1 (Gal-6S)-encoding expression plasmids were transfected individually into $\mathrm{CHO}$ cells also expressing the $\mathrm{P}$-selectin glycoprotein ligand-1/mouse immunoglobulin G2b (PSGL-1/mlg G2b) fusion protein and the human core 2 transferase (GCNT1). Characterization of the PSGL-1/mlg G2b O-glycans showed that Gal3ST2 preferentially sulfated Gal on the C-6 branch of core 2 structures and Gal3ST4 preferred Gal on the C-3 branch independently if core-1 or -2 . CHST1 sulfated Gal residues on both the C-3 (core 1/2) and C- 6 branches of core 2 structures. Using serous ovarian tissue micro array, Gal3ST2 was found to be decreased in tissue classified as malignant compared with tissues classified as benign or borderline, with the lowest expression in poorly differentiated malignant tissue. Neither Gal3ST4 nor CHST1 was differentially expressed in benign, borderline, or malignant tissue, and there was no correlation between expression level and differentiation stage. The data displays a complex sulfation pattern of $\mathrm{O}$-glycans on OC glycoproteins and that aggressiveness of the cancer is associated with a decreased expression of the Gal3ST2 transferase.
Epithelial ovarian cancer (OC) is the most lethal gynecological cancer with a $46 \%$ survival rate 5 years after diagnosis (1). The poor prognosis is related to the fact that most of the patients $(75 \%)$ get their diagnosis first in the advanced stage of the disease. Partly, this is due to the asymptomatic nature of early-stage OC. Today there are limited diagnostic and imaging tools available to distinguish between cystadenomas and cystadenocarcinomas, which prevents a correct and early diagnosis and subsequent appropriate treatment. The most frequently used marker for OC in blood is the mucin glycoprotein MUC16 (also known as CA125). CA125/MUC16 has high prognostic value for post therapy follow-up but has poor sensitivity for diagnosing ovarian carcinomas in the early stages.

The presence of mucin-type glycoproteins in ovarian tumor tissue and cyst fluid has been known for more than 70 years, and they have been shown to carry $A B O$ and Lewis blood group glycan motifs (2). Using proteomics and immunohistochemistry, we and others have shown that in addition to CA125/MUC16, there are other mucins in ovarian tumor cyst fluids, namely MUC5AC, MUC5B, MUC6, and MUC1, although their absolute and relative abundances are unknown $(3,4)$. Mucin glycosylation changes involving truncation of $O$ glycans are observed in many epithelial cancers, most likely providing cancer cells beneficial properties to spread in the body and to avoid detection by the immune system (5-9). The occurrence and potential use of cancer associated carbohydrate epitopes (Tn, sialyl Tn, T-antigen, sialyl Lewis a, and sialyl Lewis $x$ ) on CA125/MUC16 and MUC1 in serum and tissue sections from OC patients for differential diagnosis are currently being explored (10-12). Less attention has been given to the role of sulfation in $\mathrm{OC}$ tissue. Increased levels of the glycosaminoglycan chondroitin sulfate (CS), a major

\footnotetext{
From the ${ }^{1}$ Department of Medical Biochemistry and Cell Biology, ${ }^{2}$ Department of Clinical Pathology, Sahlgrenska University Hostpital, ${ }^{3}$ Department of Laboratory Medicine, Institute of Biomedicine, Sahlgrenska Academy, ${ }^{4}$ Department of Obstetrics and Gynecology, Institute of Clinical Sciences, Sahlgrenska Center for Cancer Research, University of Gothenburg, Gothenburg, Sweden; ${ }^{5}$ Department of Life Sciences and Health, Faculty of Health Sciences, Oslo Metropolitan University, Oslo, Norway

${ }^{\ddagger}$ Co-first author.

${ }^{\S}$ Co-last author.

*For correspondence: Niclas G. Karlsson, niclaska@oslomet.no.
} 
constituent of the extracellular matrix, and increased mRNA levels of CS-associated sulfotransferases, have been observed in OC [reviewed in $(13,14)]$. The limited availability of analytical tools such as antibodies or lectins used for detection and quantitation of sulfated $\mathrm{O}$ - or $\mathrm{N}$-glycans has hindered research within this field.

We have previously characterized the glycosylation on mucin-type proteins from benign and malignant mucinous and serous ovarian tumor cyst fluids using a mass spectrometric approach (15). Here, we aimed to further investigate sulfation of Gal-residues on O-glycans of OC glycoproteins, since our previous investigation indicated that this was the characteristic feature of serous cystadenoma O-linked glycosylation that distinguished it from adenocarcinoma. The elusive role of sulfated Gal-residues in cancer in general and $\mathrm{OC}$ in particular is illustrated by the OC associated HMOCC1 carbohydrate epitope, containing both 3-linked and 6linked sulfated Gal residues (16). Its role in primary cancer development is still unrevealed. The sulfated O-glycans collected from ovarian tumor cyst fluids from two patients, one diagnosed with a serous benign adenoma and one diagnosed with a low-grade serous carcinoma (LGSC), were sequenced using LC-MS ${ }^{n}$ experiments. Using recombinant expression, we tested the specificity of three Galsulfotransferase candidates on $\mathrm{O}$-glycans, and using serous ovarian tissue microarrays (TMA) probed with antibodies against sulfotransferase candidates, we addressed the question of sulfotransferase regulation during $\mathrm{OC}$ development.

\section{EXPERIMENTAL PROCEDURES}

Chemicals were from Merck, Sigma-Aldrich if not otherwise stated.

\section{Clinical Samples}

Ovarian tissue samples and cyst fluids were collected after patients signed informed consent. The collection, storage, and analysis procedures were approved by the local Ethics Committee at Sahlgrenska University Hospital (Dnr: S348-02 and S445-08) and abide by the Declaration of Helsinki principles. Ovarian tumor cyst fluids used for LC-MS ${ }^{n}$ were purified from two patients as described elsewhere (15) from one patient diagnosed with serous cystadenoma and one with a low-grade serous carcinoma (LGSC) of FIGO stage IIIC (age range of LC-MS ${ }^{n}$ analyzed patients was 55-85 with plasma CA125 8-2500 U/ $\mathrm{ml})$. Data of the patient cohort for the TMA are summarized in supplemental Table S4.

\section{Preparation of Desialylated O-glycans From Ovarian Tumor Cyst Fluids}

Acidic glycoproteins from ovarian tumor cyst fluid samples were purified as described elsewhere (15). Briefly, acidic glycoproteins were extracted from ovarian tumor cyst fluids using DEAE anion exchange chromatography followed by ethanol precipitation. Glycoproteins were resuspended in $3.5 \mathrm{M}$ urea and dot-blotted onto polyvinylidene fluoride (PVDF) membranes and stained with Direct Blue 71 . Oligosaccharides were released by reductive $\beta$-elimination from the PVDF membrane as described elsewhere (17). The reduced glycans were chemically desialylated using $2 \%$ acetic acid for 45 min at $95^{\circ} \mathrm{C}$.

Cell Culture, Expression Vectors, Transfection and Clonal Selection, Production and Purification of PSGL/mlgG2b Fusion Proteins

To evaluate the specificities of candidate sulfotransferases (CHST1, Gal3ST2, and Gal3ST4) in vitro, plasmids encoding these sulfotransferases were transiently expressed in CHO-K1 cells (ATCC) together with plasmids encoding the P-selectin glycoprotein ligand-1/ mouse immunoglobulin G2b (PSGL-1/mlgG2b) fusion protein, with or without the plasmid encoding the $\beta 1,6-N$-acetylglucosaminyltransferase 1 (GCNT1) responsible for the synthesis of core $2 \mathrm{O}$-glycans. $\mathrm{CHO}-$ $\mathrm{K} 1$ cells were cultured in 75- $\mathrm{cm}^{2}$ T-flasks (Nunc) in Dulbecco's modified Eagle's medium (DMEM, Lonza Group Ltd) supplemented with $10 \%$ fetal bovine serum (FBS, Invitrogen $A B$ ) and were transfected $24 \mathrm{~h}$ later at a cell confluence of 70 to $80 \%$. The cells were transfected using the Lipofectamine 2000 Transfection Reagent Kit (Invitrogen). Twentyfour micrograms of plasmid was used for transfection of one $75-\mathrm{cm}^{2}$ flask. Plasmids encoding human galactose-3-O-sulfotransferase 2 and 4 mRNA (pEZ-M67-Gal3ST2 and pEZ-M67-Gal3ST4) were obtained from GeneCopoeia. The human keratan sulfate Gal-6-sulfotransferase (CHST1) was amplified by PCR from a human placenta CDNA library using forward (5'-CGCGGGAAGCTTACCATGCAATGTTCCTGGAA GG-3') and reverse (5'-CGCGGCGGCCGCTCACGAGAAGGGGCG GAAGTC-3') primers and was swapped into a CDM 8-based vector carrying the CMV promoter using Hind III/Not I restriction sites. The plasmids harboring PSGL-1/mlgG2b and GCNT1 were constructed as described previously (18). After an 8-day incubation in $15 \mathrm{ml}$ DMEM with $10 \%$ FBS, the supernatant was collected and cells were removed by centrifugation at $5020 \mathrm{~g}$ for $30 \mathrm{~min}$. The different recombinant PSGL-1/mlgG2b fusion proteins were purified from $15 \mathrm{ml}$ of supernatant by mixing with $30 \mu \mathrm{l}$ of goat anti-mouse IgG agarose beads in a tube rotated at $4{ }^{\circ} \mathrm{C}$ for $6 \mathrm{~h}$. The beads were washed two times with phosphate buffered saline (PBS) and boiled in LDS nonreducing sample buffer ( $30 \mu \mathrm{l}$, Invitrogen AB) before SDS-PAGE electrophoresis and $O$-glycan analysis. In brief, recombinant proteins were separated on 3 to $8 \%$ NuPAGE gels (Invitrogen $A B$ ) and electrophoretically blotted onto PVDF membrane (Immobilon P membranes, Millipore) using a semidry method. O-glycans were released from PVDF membrane stripes using reductive $\beta$-elimination as described previously $(17,19)$

\section{LC-MS of O-glycans and Data Assignments}

For LC-MS, the glycans were separated on porous graphitized carbon columns (PGC, $5 \mu \mathrm{m}$ particles, Hypercarb, Thermo Fisher Scientific) prepared in-house and with $10 \mathrm{~cm}$ length and $250 \mu \mathrm{m}$ inner diameter. The gradient was 0 to $40 \%$ acetonitrile containing $10 \mathrm{mM}$ ammonium bicarbonate over $60 \mathrm{~min}$, kept at a flow rate of 5 to $10 \mu \mathrm{l} / \mathrm{min}$. Mass spectrometric data was collected at low resolution in the negative ion mode using a Thermo Scientific LTQ ion trap mass spectrometer.

MS (collision-induced dissociation) analyses of $\mathrm{O}$-glycans were performed by repeated injections of the same sample and analyzed with data-dependent $\mathrm{MS}^{n}$ experiments. The glycans were detected as deprotonated $\left([\mathrm{M}-\mathrm{nH}]^{\mathrm{n}-}\right)$ ions. For the $\mathrm{LC}$ runs involving $\mathrm{MS}^{2}$ experiments, $\mathrm{MS}^{2}$ was performed on the six most abundant ions in every full scan or performed throughout the run on a predetermined ion of interest. For LC runs involving $\mathrm{MS}^{3}$ and $\mathrm{MS}^{4}$ analyses (ovarian tumor cyst fluid glycans), data-dependent MS experiments were performed where each target ion was continuously selected for fragmentation throughout the whole run (summarized in supplemental Table S3). Constant parameters were electrospray voltage $3.5 \mathrm{kV}$, capillary voltage of $-6 \mathrm{~V}$, and capillary temperature of $300{ }^{\circ} \mathrm{C}$. Full MS scans 
were collected at $\mathrm{m} / \mathrm{z} 380$ to 2000 with an isolation width of $\mathrm{m} / \mathrm{z} 2$ and normalized collision energy of $35 \%$.

MS spectra were interpreted manually, and the assignment of sequences and annotation of fragment ions were confirmed using freely available software "GlycoWorkbench" (20). Reference spectra for sulfated core 1 sequences were collected from PSGL-1/mlgG2b purified from supernatants of transient $\mathrm{CHO}-\mathrm{K} 1$ transfectants expressing sulfotransferases Gal3ST4, CHST1, and PSGL1/mlgG2b without cotransfection with GCNT1 (see section on recombinant proteins). The following assumptions were made based upon known features of mucin-type O-glycosylation: Hex residues are Gal, HexNAc are GlcNAc, reducing-end sugar is GalNAc-ol.

\section{Tissue Micro Array (TMA) and Scoring}

Sulfotransferase protein expression levels in serous ovarian tissues were evaluated using TMA with tissue from 323 ovarian tumor specimens (supplemental Table S4). The construction and scanning of the TMA are described elsewhere (21). The paraffin sections were deparaffinated in xylene and then rehydrated. Tris-base $\mathrm{pH} 9$ was used as unmasking solution. The sections were counterstained with Hematoxylin.

The tissue sections were incubated with polyclonal antibodies produced in rabbit: Anti-CHST1 (ARP45495_T100, Aviva Systems Biology), anti-Gal3ST2 or anti-Gal3ST4 (HPA071809 and HPA038137, Prestige Antibodies supported by Human Protein Atlas, SigmaAldrich). The antibody dilutions were for CHST1, 1:100; for Gal3ST2, 1:20; and for Gal3ST4, 1:50 in PBS. They were incubated with the tissue for $90 \mathrm{~min}$. Antibody binding was measured using UltraVision Quanto Detection System AP (Thermo Scientific) according to the protocol.

A semiquantitative method "quick score" (QS) for manual evaluation of tissue staining was applied. Proportion of stained tissue was graded 0 to 4 , based upon percentage of stained tissue within the following intervals: $0=0 \%, 1=1$ to $25 \%, 2=26$ to $50 \%, 3=51$ to $75 \%$, and $4=76$ to $100 \%$. Intensity of the stain was graded 0 to 3 (negative, weak, moderate, and strong). The scores for proportion and intensity were summed, generating a maximum score value of 7 . Every tissue section was scored blindly by pathologist (CM) in duplicates or triplicates.

\section{Experimental Design and Statistical Rationale}

Chemical desialylation of oligosaccharides from cyst fluids from two patients with benign and malignant epithelial ovarial tumors was performed once due to limited availability. These samples were injected 17 and 14 times (benign and malignant, respectively) each for the targeted $\mathrm{MS}^{\mathrm{n}}$ experiments outlined in supplemental Table S3. The OC MS data presented in this paper is primarily presented as descriptive. For the study of the specificity of sulfotransferases on recombinantly produced PSGL1/mlgG2b, the data presented here were generated from one transfection with sulfotransferase Gal3ST2 or two transfections performed with CHST1 and Gal3ST4. Glycosylation from each transfection was analyzed with LC-MS once. TMA sulfotransferase staining and intensity scoring were performed once on the full sample set due to limited availability and validated on a subset $(n=28)$ of serous OC tissue on a separate TMA. QS was used to assess the level of staining in order to identify differences in expression depending on pathological assessment of malignancy and the level of differentiation of malignant cases. Significance of the differences in expression was calculated using multiple comparison between the different categories of malignancy and differentiation level using Kruskal-Wallis test using its module in Prism 8.4.2 (Graphpad Softwares Inc). Human colon and placenta tissue slides were used as positive controls according to their expression recorded in the human proteome atlas (www.proteinatlas.org/).
RESULTS

Analyses of Desialylated Sulfated Oligosaccharides From Serous Ovarian Tumor Cyst Fluids

Oligosaccharides were released by reductive $\beta$-elimination from enriched acidic proteins from cyst fluids from two patients, one patient diagnosed with benign tumor and one diagnosed with malignant LGSC. The oligosaccharides were desialylated to aid MS identification of sulfate location. The Oglycans were subjected to analyses with LC-MS $^{n}$ using porous graphitized carbon chromatography and negative ion mode. Base peak chromatograms are displayed in Figure 1, $A$ and $B$, where major peaks are assigned. The LC-MS profiles confirmed that sulfated glycans were abundant in these samples. The degree of sialylation before removal of these residues is compiled in supplemental Table S2 and also described within a previous study (15). Major sulfated glycan precursor ions were identified, followed by repeated analyses of the samples using data-dependent $\mathrm{MS}^{n}$ experiments to ensure sufficient number of $\mathrm{MS}^{2}, \mathrm{MS}^{3}$, and $\mathrm{MS}^{4}$ spectra on the low-abundant sulfated compounds. Fifty-four sulfated glycans consisting of 2 to 10 monosaccharide residues with 1 to 4 sulfate residues were detected, many as isomeric structures. The general structural theme of identified oligosaccharides is shown in Figure $1 C$, and the compiled list of deduced sequences is found in supplemental Table S1.

Core 1 Glycans From Serous Ovarian Tumor Cyst Fluids

Carry Sulfate Linked to Hydroxyl Groups at C-3 and C-6 of Gal

Two sulfated core 1 type glycans were detected in the serous ovarian tumor cyst fluid samples, two monosulfated disaccharides ( $\mathrm{HSO}_{3}$-Galp1-3GalNAcol) detected as $[\mathrm{M}-\mathrm{H}]^{-}$ions at $m / z$ ab4 labeled "464-1" and "464-2" (supplemental Table S1). The extracted ion chromatograms of $\mathrm{m} / \mathrm{z} 464$ from the two patients are inserted in Figure $2 B$, highlighting that the component retention times and the relative abundances were similar in both samples. The corresponding $\mathrm{MS}^{2}$ spectra were identical in the two samples, supporting that the same glycans were present. MS $^{2}$ spectra of " $464-1$ " and "464-2" from the serous benign tumor cyst fluids are shown in Figure 2, $A$ and $B$. Both spectra contained an intense $C$ type fragment ions at $m / z 241^{-}$, supportive of sulfate predominantly linked to the Gal residue, and the late eluting sequence "4641" displayed low intense Gal-cross ring fragment ions at $m / z$ $199^{-}\left({ }^{0,2} \mathrm{~A}\right)$ shown previously to be formed when sulfate is linked via C-6 to Gal (22). The two glycoforms were deduced as " $\mathrm{HSO}_{3}-3 \mathrm{Gal} \beta 1-3 \mathrm{GalNAcol}$ " ("464-1") and " $\mathrm{HSO}_{3}-6 \mathrm{Gal} \beta 1$ 3GalNAcol" ("464-1") by comparison to $\mathrm{MS}^{2}$ spectra of reference compounds, obtained from recombinantly produced PSGL1/mlgG2b fusion protein transfected into $\mathrm{CHO}$-cells together with sulfotransferases CHST1 or Gal3ST4. (supplemental Fig. S1).

Evidence for a third, lower-abundant glycoform coeluting with "464-1" (Fig. 2A) and present in the samples from both 


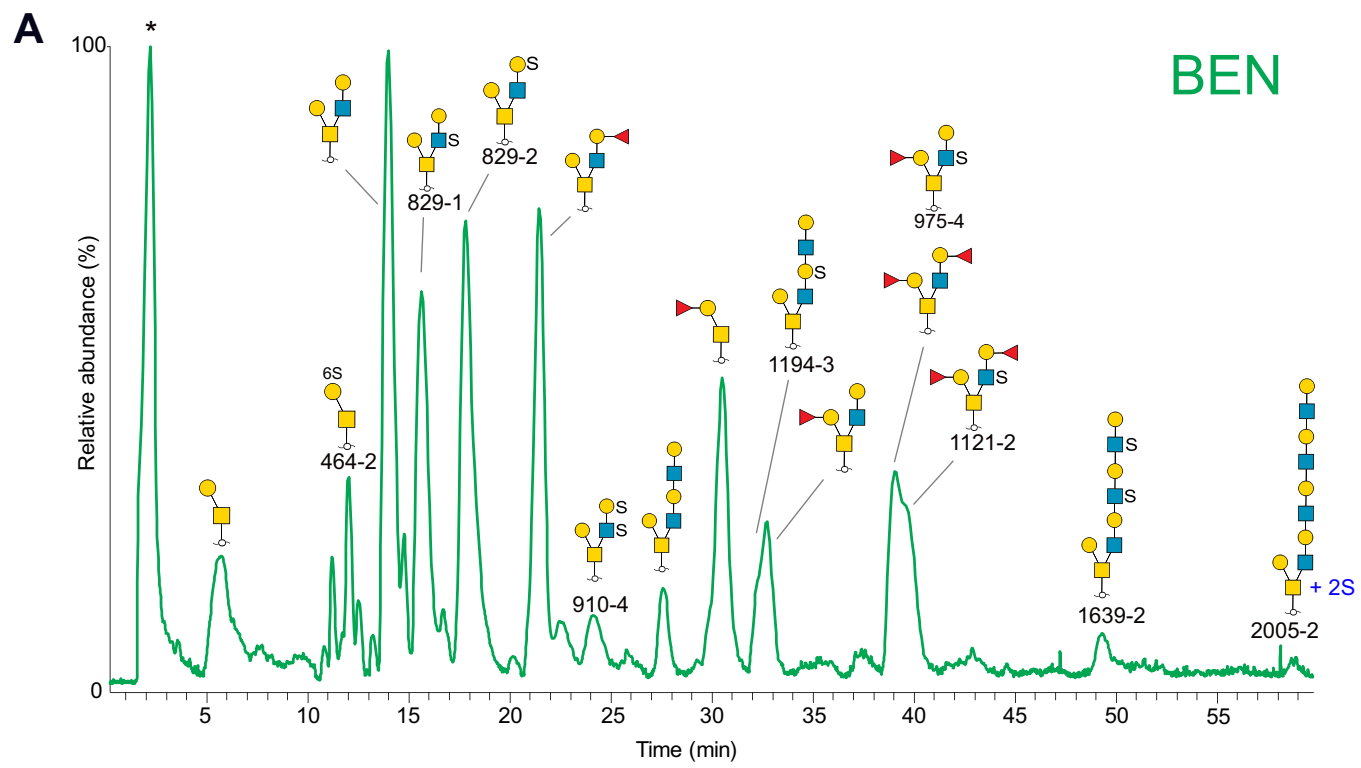

B

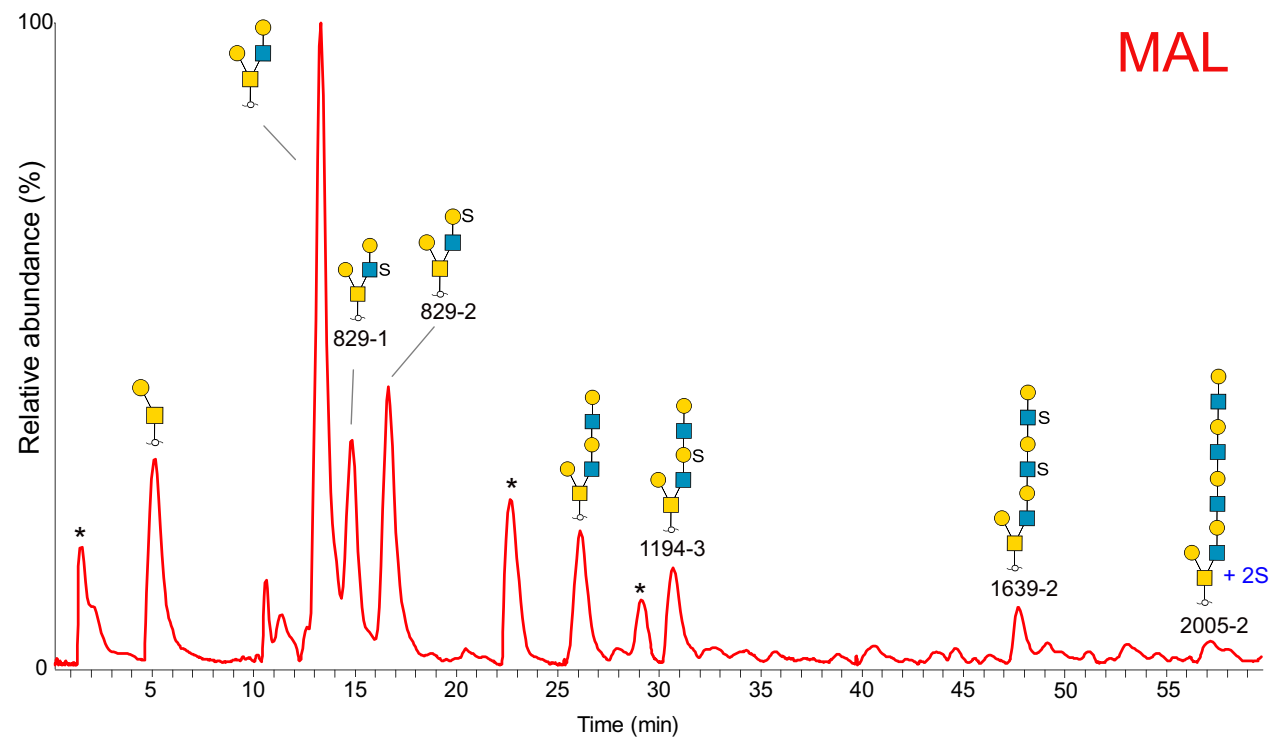

C

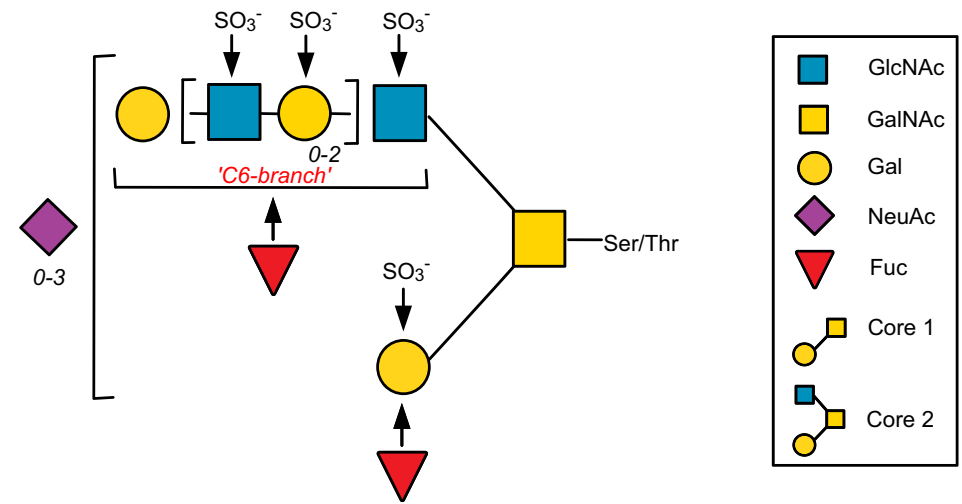

FIG. 1. LC-MS base peak chromatograms of desialylated sulfated glycoforms, purified from serous ovarian tumor cyst fluids from patients with serous benign and malignant tumor. $A$, beningn tumor ("BEN") and $B$, malignant tumor ("MAL"). Major glycans are annotated using SNFG nomenclature. Sulfated glycans are annotated with name in black font and are listed in supplemental Table S1. Contaminants are 


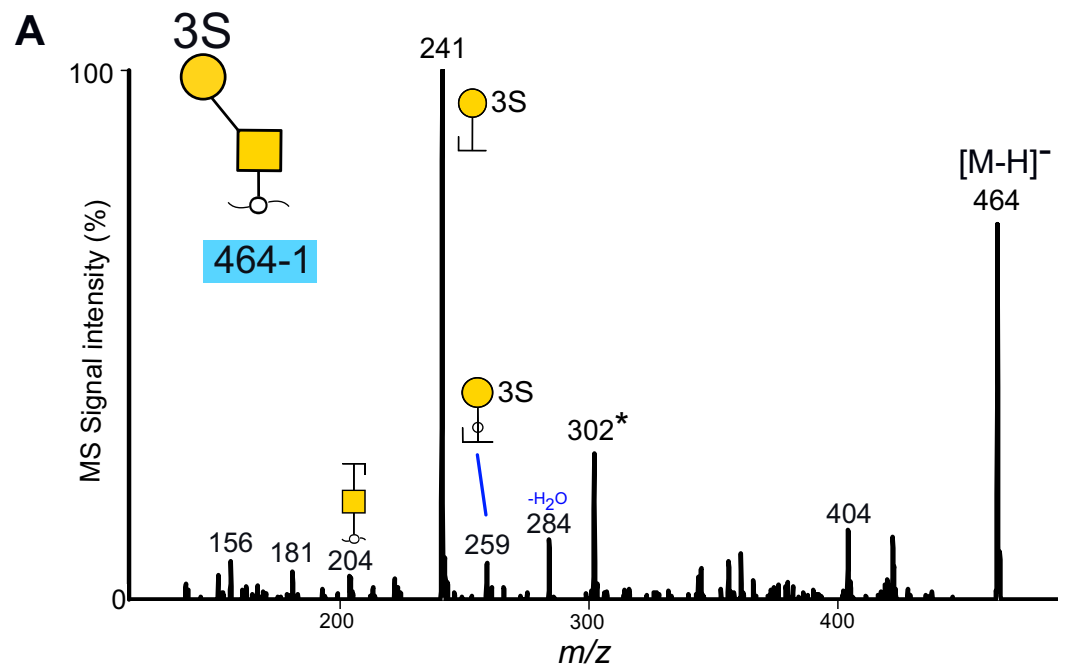

B

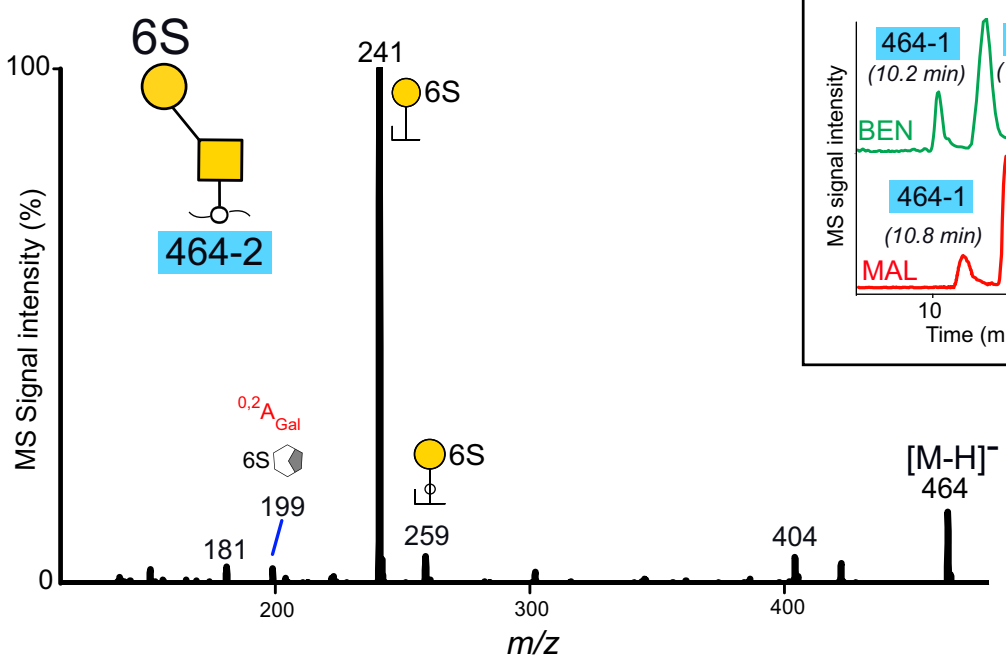

FIG. 2. $\mathrm{MS}^{2}$ spectra of sulfated core 1 disaccharides at $\mathrm{m} / \mathrm{z} 464([\mathrm{M}-\mathrm{H}]]^{-}$ions)purified from cyst fluids from $\mathrm{OC}$ patients with benign ("BEN") and malignant ("MAL") tumors. Components are labeled as 464-1 (A) and 464-2 (B). The extracted ion chromatograms from the full scans are inserted. For key to symbols, see Figure 1. ${ }^{*}$ The ions at $m / z 302$ in panel $A$ correspond to a fragment ion consisting of sulfate linked to the core reducing sugar GalNAcol and may originate from minor amounts of a coeluting glycoform.

the benign and the malignant tumor cyst fluids, where a fragment ion at $\mathrm{m} / \mathrm{z} 302$ indicative of sulfate linked to the core GalNAcol was observed (23).

\section{Core 2 Glycans From Serous Benign and Malignant Tumor} Cyst Fluids Have Sulfate Linked to Both GlcNAc and Gal Residues

The majority of the desialylated glycans detected from cyst fluids were assigned as core 2 type with a single Gal residue linked via C-3 to GalNAcol and an extended C6 branch (Fig. 1C). Examples of two short, sulfated saccharides are displayed in Figure 3. Extracted ion chromatograms of two abundant tetrasaccharides detected at $m / z 829\left([\mathrm{M}-\mathrm{H}]^{-}\right.$precursor ions) from the two patients are inserted in the figure. The two components that were labeled "829-1" and "829-2" had similar retention times, relative abundances, and the same MS spectra in both samples. Both glycans were interpreted as core 2 sequences, where the most intense fragment ions corresponded to loss of a Hex residue $\left(\mathrm{m} / \mathrm{z} 667^{-}\right)$. The ions at $\mathrm{m} / \mathrm{z} 444^{-}$in both spectra were indicative of a branch consisting of Hex, HexNAc, and sulfate. The ions at $\mathrm{m} / z 505^{-}$and 282 $2^{-}$in 829-1 (Fig. 3A) supported a core 2 sequence with sulfate linked to HexNAc and thus interpreted as Gal-3(Gal$\left(\mathrm{HSO}_{3}{ }^{-}\right)$GlcNAc-6)GalNAcol. Component "829-2" (Fig. 3B)

labeled with *. In $C$, general structural theme of sulfated $O$-glycans from serous ovarian tumor cyst fluids is displayed. The GalNAc residue is linked to serine or threonine in the protein. Residues marked with arrows indicate identified sites for fucosylation or sulfation. Numbers in italics indicate that Gal-GlcNAc (highlighted in brackets) and NeuAc residues can occur 0, 1, 2, and 3 times in the molecule. Symbol key is according to the SNFG nomenclature for glycans (36). 
A

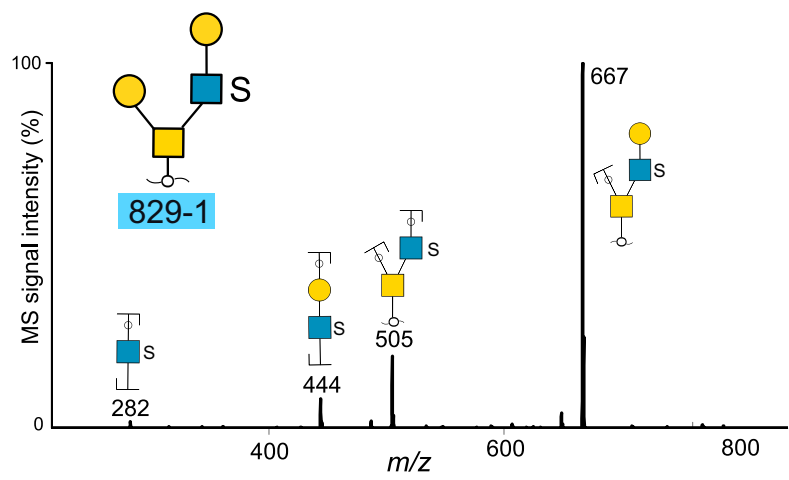

B
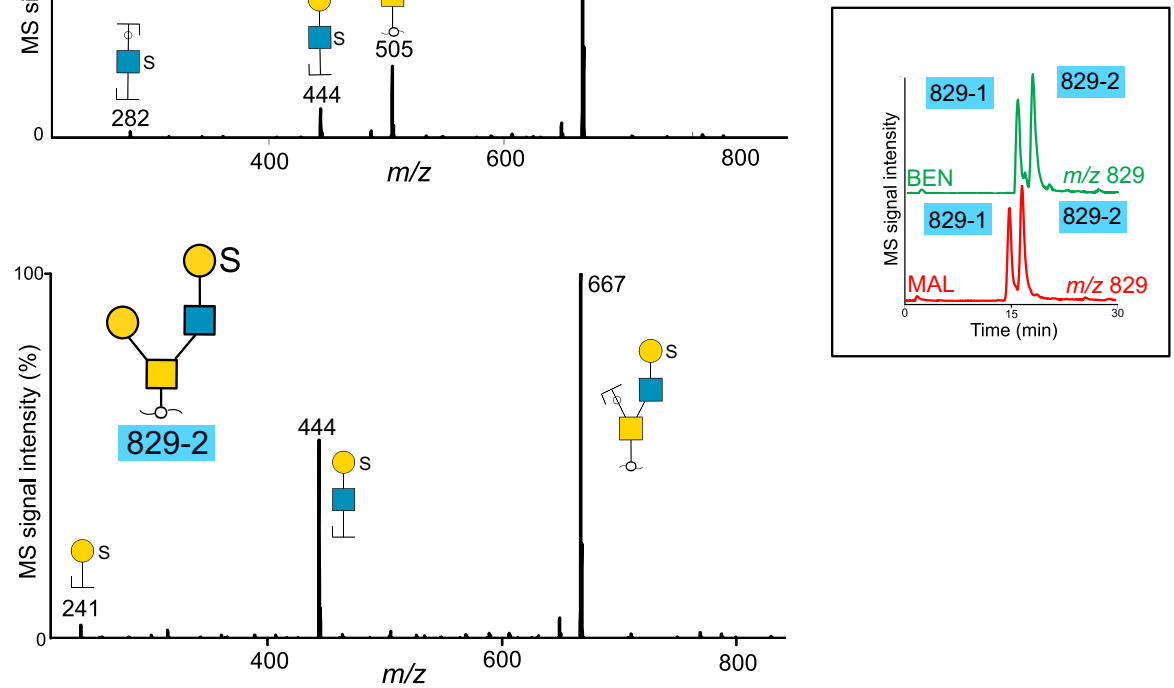

FIG. 3. $\mathbf{M S}^{2}$ spectra of desialylated sulfated core 2 tetrasaccharides detected at $\mathbf{m} / \mathbf{z} \mathbf{8 2 9}$ ( $\mathbf{M}^{-}$ions) purified from cyst fluids from two patients with benign ("BEN") and malignant ("MAL") tumors. Components are labeled as 829-1 (A) and 829-2 (B). The extracted ion chromatograms are inserted. For key to symbols, see Figure 1.

contained fragment ions at $\mathrm{m} / \mathrm{z} 241^{-}$, indicative of sulfate

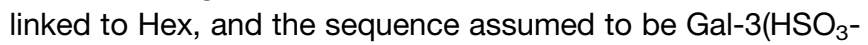
Gal-GlcNAc-6)GalNAcol. The spectra did not reveal fragment ions, which enabled assignment of C-3 or C-6 linked sulfate. Additional identified structures also showed sulfation either on Gal and GlcNAc residues or both (supplemental Table S1).

Data-dependent MS ${ }^{n}$ Experiments Revealed Doubly, Triply, and Tetrasulfated Glycans With a Single Hex Linked on the Core 2 C-3 Branch and Extended LacNAc Chain Linked to the C-6 Branch

A general structural theme of the core 2 type sulfated oligosaccharides was found in cyst fluids from the two patients with benign and malignant tumors, with the glycan chain extension restricted to the C-6 arm and consisting of up to four HexHexNAc units (Fig. $1 C$ and supplemental Table S1). Increasing number of sulfates caused the glycans to bind strongly to the porous graphitized carbon (PGC) column. The largest glycans that were detected here consisted of five Hex, five HexNAc, and two sulfate residues ("2005-1, -2, and -3," supplemental Table S1). These eluted within a minute before the gradient ended, precluding detection of larger glycans eluting later.

In many cases the sulfate residues could be assigned to inner Gal or GlcNAc residues in the glycan. This was supported by the detection of an abundant fragment ion in $\mathrm{MS}^{2}$ spectra generated from the loss of a terminal nonsulfated Hex, followed by a second
$\mathrm{MS}^{3}$ collision experiment of this ion, displaying the loss of a second, nonsulfated Hex. Most of these compositions were identified to contain sialic acid before desialylation (supplemental Table S2), suggesting that terminal Hex residues are sialylated. An example of the type of structure found is illustrated in Figure 4. Figure $4 A$ shows the base peak chromatogram of the sulfated glycoforms detected at $\mathrm{m} / \mathrm{z} 819^{2-}$ / $1639^{-}$, originating in malignant cyst fluid proteins. The monosaccharide composition corresponded to oligosaccharides consisting of eight residues and two sulfate groups. The deduced sequences in Figure $4 A$ are listed in supplemental Table S1. All were found to be of core 2 type, with a single nonsulfated Hex linked to the reducing HexNAcol, and a second extended disulfated triLacNAc chain carrying sulfate in different positions causing the observed structural variety. The complexity is illustrated by at least five detected chromatographic peaks in Figure 4A, labeled "1639-1" to "1639-5." The most abundant component ("1639-2") was found to be sulfated on the two outer HexNAc residues in the triLacNAc unit. Of these "1639" isomers, the "1639-2" was the dominating isomer detected in the sample purified from the patient with a benign tumor (supplemental Table S1). Figure $4 B$ displays $\mathrm{MS}^{2}$ spectra of one of the minor components labeled "1639-3" only present in the malignant sample. The major fragment ions at $m / z 738.1^{2-}$ originated from loss of a terminal Hex residue. It is surrounded by singly and doubly charged fragment ions from the extended 


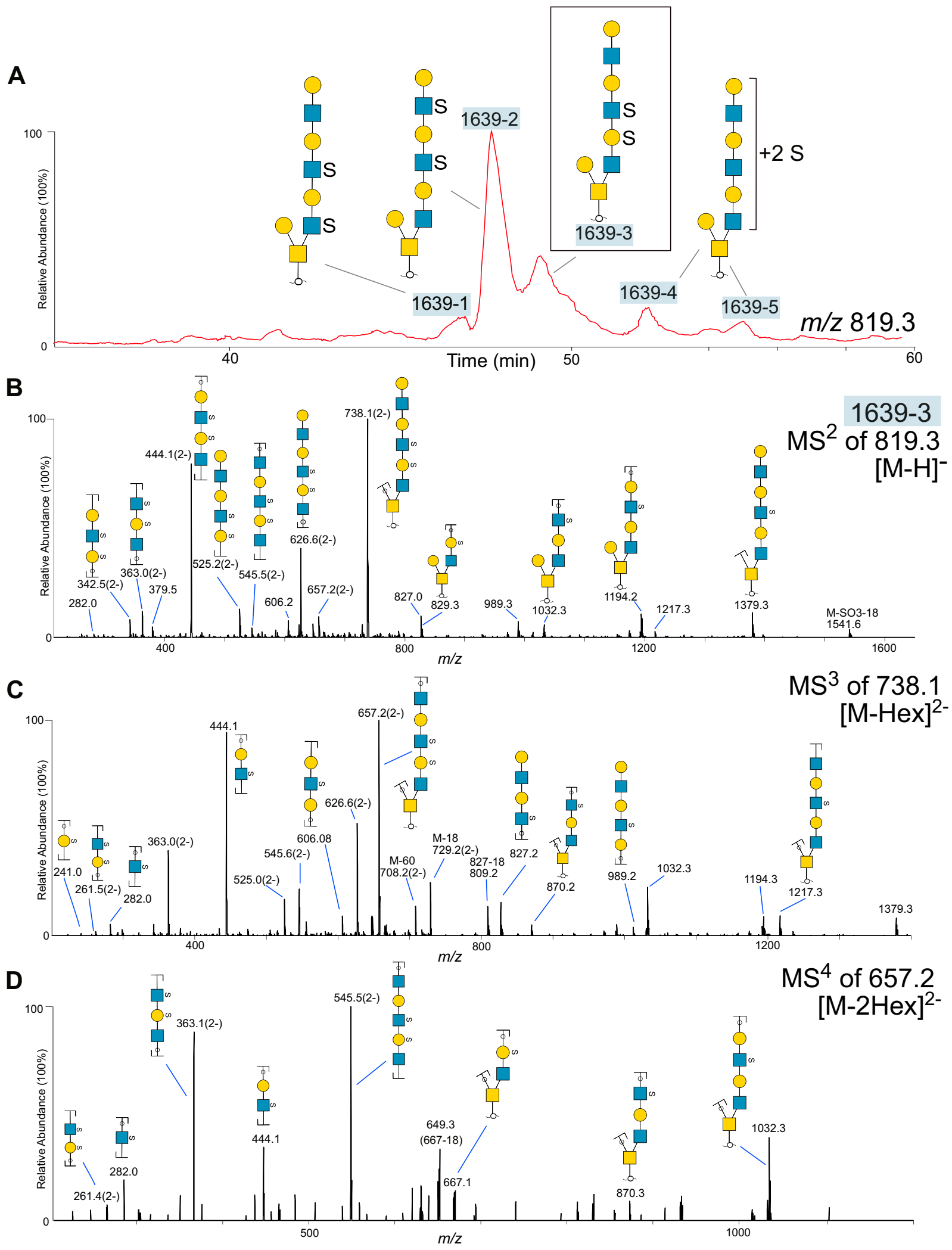

FIG. 4. LC-MS ${ }^{\mathbf{n}}$ detection and characterization of disulfated octasaccharides in ovarian cyst fluid. $A$, extracted ion chromatograms of desialylated disulfated oligosaccharides detected at $\mathrm{m} / \mathrm{z} 819^{-}$, purified from cyst fluid from a patient with a malignant ("MAL") tumor. The parent ions were detected as $[\mathrm{M}-2 \mathrm{H}]^{2-}$ ions. Glycan sequences are interpreted from $\mathrm{MS}^{2}, \mathrm{MS}^{3}$, and $\mathrm{MS}^{4}$ experiments. $B, \mathrm{MS}^{2}$ spectra of the glycan labeled "1639-3". Fragment ions are annotated with proposed compositions. For some fragment ions, more than one composition may be possible. $C, \mathrm{MS}^{3}$ spectra of the ions at $m / z 738.1^{2-}$ from the $\mathrm{MS}^{2}$ experiment shown in $(B) . D, \mathrm{MS}^{4}$ spectra of the ions at $\mathrm{m} / \mathrm{z} 657.2^{2-}$ from the $\mathrm{MS}^{3}$ experiment shown in $(C)$. For key to symbols, see Figure 1. 
branch (B/C-ions) on the C-6 arm or containing the reducing sugar (Y/Z-ions). The presence of intense $\mathrm{C}$-ions at $\mathrm{m} / \mathrm{z} 626.6^{2-}$ confirmed the presence of the $\mathrm{C} 6$ branch, consisting of six monosaccharide residues and the two sulfates. Figure $4 C$ shows the $\mathrm{MS}^{3}$ spectra for component "1639-3" collected by the datadependent MS experiment covering the transition "819.3"- $\rightarrow$ $738.1^{2-"}\left([\mathrm{M}-\mathrm{Hex}]^{2-}\right)$. The most abundant fragment ions at $\mathrm{m} / \mathrm{z}$ $657.2^{2-}$ were indicative of loss of a second, nonsulfated, terminal Hex residue from the precursor molecule. The detection of sulfated fragment ions at $\mathrm{m} / \mathrm{z} 241^{-}, 282^{-}$, and $261.5^{2-}$ supported that those sulfates were linked to a Hex and a HexNAc, respectively, and that these saccharide residues had to be adjacent to each other. Assignment of the disulfated HexHexNAc unit within the linear chain was aided by detection of doubly charged B-ions at $m / z 525.2^{2-}$, which indicated that this unit had to be part of the five outer monosaccharide residues of the extended C- 6 branch. Detection of Y-ions at $\mathrm{m} / \mathrm{z} 829^{-}$and $870^{-}$in the $\mathrm{MS}^{2}$ and $\mathrm{MS}^{3}$ spectra confirmed that one sulfate was present on the first Hex closest to the reducing sugar in the linear C6-branch. Figure $4 D$ displays $\mathrm{MS}^{4}$ spectra covering the transition "819.3 $3^{2-} \rightarrow 738.1^{2-} \rightarrow 657.2^{2-"}\left([\mathrm{M}-2 \mathrm{Hex}]^{2-}\right)$, thus fragmenting the precursor oligosaccharide at $657.2^{2-}$, which has already lost two Hex residues. These spectra confirmed our interpretation of the sequence, since all the detected fragment ions could be deduced from the remaining residues.

Assignment of sulfate residues along the extended C6-chain of the sulfated glycans was aided by the detection of abundant doubly charged fragment ions carrying two sulfate groups, such as $m / z 261^{2-}$ consisting of two sulfates and a Hex-HexNAc unit (B-ions), and $\mathrm{m} / \mathrm{z} 373^{2-}$, doubly sulfated Z-ions consisting of Hex-HexNAc and the reducing sugar HexNAcol. A confirmation of these diagnostic ions was provided in $\mathrm{MS}^{2}, \mathrm{MS}^{3}$, and $\mathrm{MS}^{4}$ experiments of the disulfated tetrasaccharide labeled "910-4" (supplemental Table S1). This glycan was interpreted as Gal$3\left(\mathrm{HSO}_{3}-\mathrm{Gal}-\left(\mathrm{HSO}_{3}-\right)\right.$ GlcNAc-6)GalNAcol, and the $\mathrm{MS}^{\mathrm{n}}$ spectra are shown in supplemental Fig. S2. The doubly charged fragment ions at $\mathrm{m} / \mathrm{z} 373^{2-}$ were the most abundant ions in the $\mathrm{MS}^{2}$ spectra and were further collided in an $\mathrm{MS}^{3}$ experiment, revealing fragment ions at $m / z 261^{2-}$, supporting that the sulfate groups were present on Hex-HexNAc. B-ions at $m / z 241^{-}$confirmed that one sulfate was linked to a $\mathrm{Hex}$, and $\mathrm{Y} / \mathrm{Y}$-ions at $\mathrm{m} / \mathrm{z} 505^{-}$that the second sulfate was linked to a HexNAc. $\mathrm{MS}^{4}$ of $m / z 261^{2-}$ as a precursor revealed fragment ions confirming both the saccharide and sulfate constituents of this fragment.

The Pattern of Desialylated, Sulfated O-glycans From Serous Benign and Malignant Ovarian Tumor Cyst Fluids Revealed No Major Differences Regarding Nonfucosylated Sulfated Structures

Figure 5 shows the extracted MS base peak chromatograms of six different glycan compositions, consisting of between four and eight monosaccharide residues and 2 to 3 sulfate residues. The deduced compositions are shown above and are also found listed in supplemental Table S1. Overall, the same number of peaks and approximate relative abundance are observed in both samples, with one exception. One of the two glycoforms of the trisulfated tetrasaccharides shown in panel $989^{-}$is not detected in the cyst fluid sample from the patient with a malignant tumor. However, in all, these glycan profiles do not point toward any major differences regarding relative abundances of sulfated glycans from the benign and malignant cyst fluid samples, although minor differences are observed, which could be associated with altered sulfotransferase activity.

Fucose-containing Sulfated Glycans Provide Evidence for Sulfated Lewis b/y and Blood Group $H$ Sequences in Serous Benign Cyst Fluid but Only Lewis a/x Sequences in Malignant Cyst Fluid

Desialylation of serous cyst $O$-glycans also enabled structural characterization of some of the low-abundant fucoseand sulfate-containing glycans present on serous cyst fluid proteins. Figure 6 displays the base peak chromatograms of both a nonsulfated and a sulfated fucosylated pentasaccharide (panels 895 and 975) from benign and malignant cyst fluids. Sequence elucidation confirmed that the detected structures were both of blood group $\mathrm{H}$ (Fuc 1 1-2Gal-) and Lewis (Gal $\beta 1-3 / 4$ (Fuc $\alpha 1-4 / 3$ )GlcNAc-) type. The base peak chromatogram (Panel 1121) of the difucosylated sulfated hexasaccharide revealed two glycoforms detected in the benign but not in the malignant cyst sample. The glycoform labeled "1121-1" was of a Lewis b/y type (Fuc $\alpha 1-2 \mathrm{Gal} \beta 1-3$ / 4(Fuc $\alpha 1-4 / 3)$ GlcNAc-), and the corresponding $M^{2}$ spectra of the precursor ions at $\mathrm{m} / \mathrm{z} 1121\left([\mathrm{M}-\mathrm{H}]^{1-}\right)$ showed multiple diagnostic and intense fragment ions used for sequence identification. The B-ions at $\mathrm{m} / \mathrm{z} 736.1^{-}$indicated the presence of a branch containing two Fuc, sulfate, Hex, and HexNAc, and the $Y$-ion at $m / z 959^{-}$, formed by loss of a nonsubstituted, terminal Hex residue. The fragment ions at $\mathrm{m} / \mathrm{z} 505^{-}$confirmed that sulfate was present on the HexNAc adjacent to the reducing sugar, and the ions at $\mathrm{m} / \mathrm{z} 428^{-}$were diagnostic of a HexNAc substituted with both sulfate and Fuc. The sequence was thus deduced as equivalent to a core 2 Lewis $b$ or Lewis $y$ sequence: $\quad$ Gal $\beta 1-3$ (Fuc $\alpha 1-2 \mathrm{Gal} \beta 1-3 / 4$ (Fuc $\alpha 1-4 / 3)(\mathrm{HSO} 3-)$ GlcNAc $\beta 1-6)$ GalNAc.

Sulfotransferases CHST1, Gal3ST2, and Gal3ST4 Add Sulfate to C-3 and C-6 of Different Gal Residues in Core 2 O-glycans on PSGL-1/mlgG2b Fusion Proteins

Our results highlighted that sulfate linked to Gal residues both within and at the terminal nonreducing end of the $O$ glycan branches is a significant feature of ovarian glycoproteins. In order to test if some of the identified sulfotransferases described in the literature were able to use mucin core 1 and/ or $2 \mathrm{O}$-glycans as targets, PSGL-1/mlgG2b was co-expressed with the core 2 glycosyltransferase (GCNT1) and the Gal sulfotransferases Gal3ST2, Gal3ST4, or CHST1 in CHO cells. The three sulfotransferases have been shown to add sulfate to $\mathrm{C}-3$ 


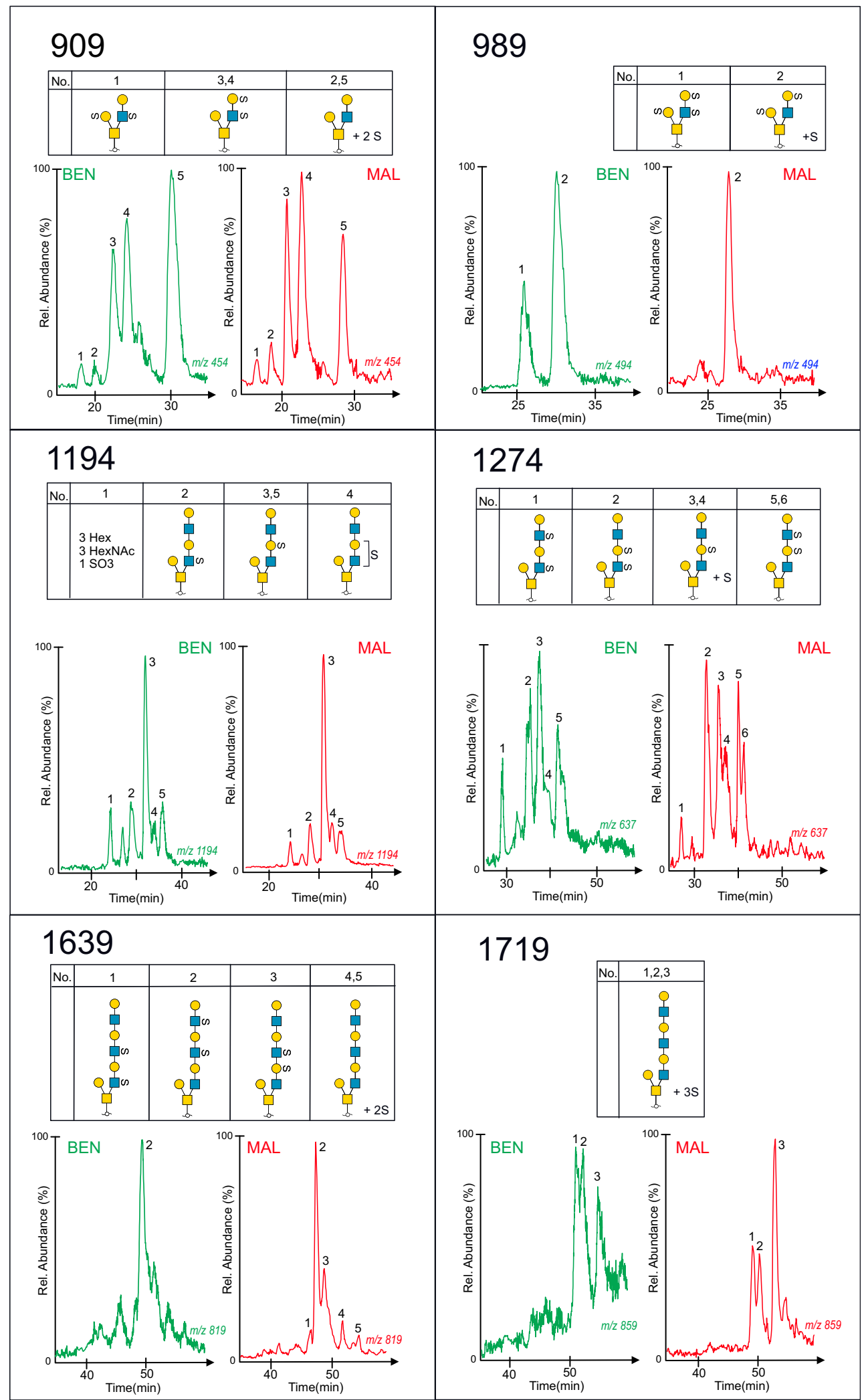

FIG. 5. Extracted ion chromatograms of desialylated sulfated glycoforms, purified from serous cyst fluids from OC patients with benign (labeled in green) and malignant (labeled in red) tumors. Glycan sequences were interpreted from $\mathrm{MS}^{2}, \mathrm{MS}^{3}$, and $\mathrm{MS}^{4}$ experiments. Glycan panel names $(909,989,1194,1274,1639$, and 1719) correspond to their mass in the deprotonated form and are listed in supplemental Table S1. For key to symbols, see Figure 1. 


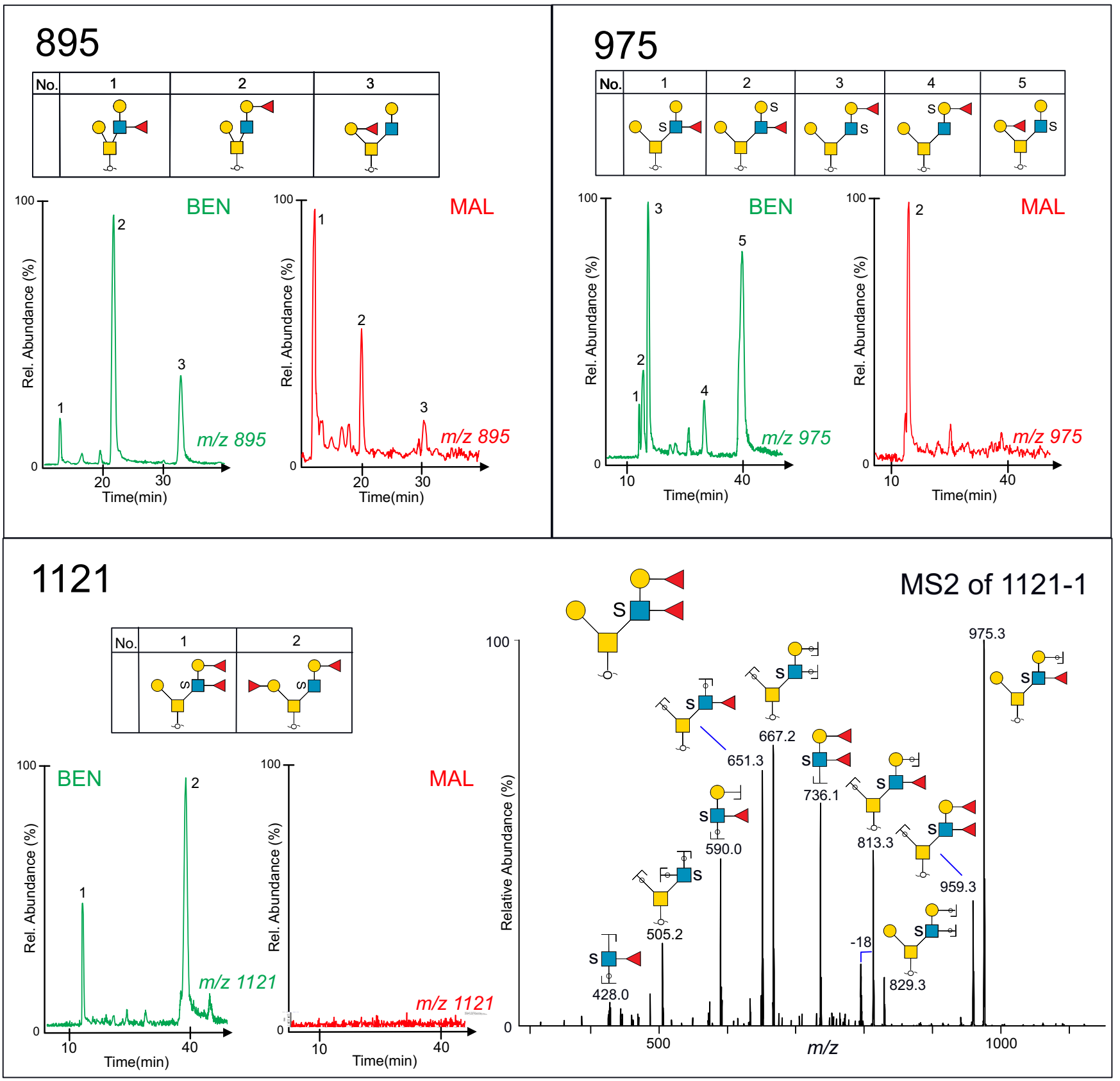

FIG. 6. Extracted ion chromatograms of desialylated fucose- and sulfate-containing glycoforms, purified from cyst fluids from benign (labeled in green) and malignant ovarian tumors (labeled in red). Glycan sequences were interpreted from $\mathrm{MS}^{2}$ experiments. Glycan panel names $(895,975,1121)$ correspond to their mass in the deprotonated form and are listed in supplemental Table S1. MS ${ }^{2}$ of a sulfated Lewis b or Lewis y type glycan "1121-1" detected as $m / z 1121\left([\mathrm{M}-\mathrm{H}]^{-}\right.$precursor ion) is displayed in the lower panel. For key to symbols, see Figure 1.

(Gal3ST2 and Gal3ST4) and C-6 (CHST1) of Gal. We have previously published the O-glycosylation of PSGL-1/mlgG2b expressed in $\mathrm{CHO}$ cells together with the core 2 GCNT1 but without sulfotransferases, and the glycans were found to be nonsialylated, mono- and disialylated core 1 and core 2 glycans consisting of 3 to 6 monosaccharide residues (18). PSGL-1/mlgG2b from the three transfections was purified, the $O$-glycans released using reductive $\beta$-elimination, followed by analyses with LC-MS and $\mathrm{MS}^{2}$ experiments. Interpretations are compiled in supplemental Fig. S3. The extracted ion chromatograms and $\mathrm{MS}^{2}$ spectra of the core 2 tetrasaccharide with the sulfate added to the sequence Gal $\beta 1$ 3(Gal $\beta 1-4 G l c N A c \beta 1-6)$ GalNAcol detected as $\mathrm{m} / \mathrm{z} 829^{-}\left([\mathrm{M}-\mathrm{H}]^{-}\right.$ ions) are shown in Figure 7. All three sulfotransferases generated only one glycoform product each. All three $\mathrm{MS}^{2}$ spectra revealed fragments ion at $\mathrm{m} / \mathrm{z} 241^{-}$, diagnostic of 
A

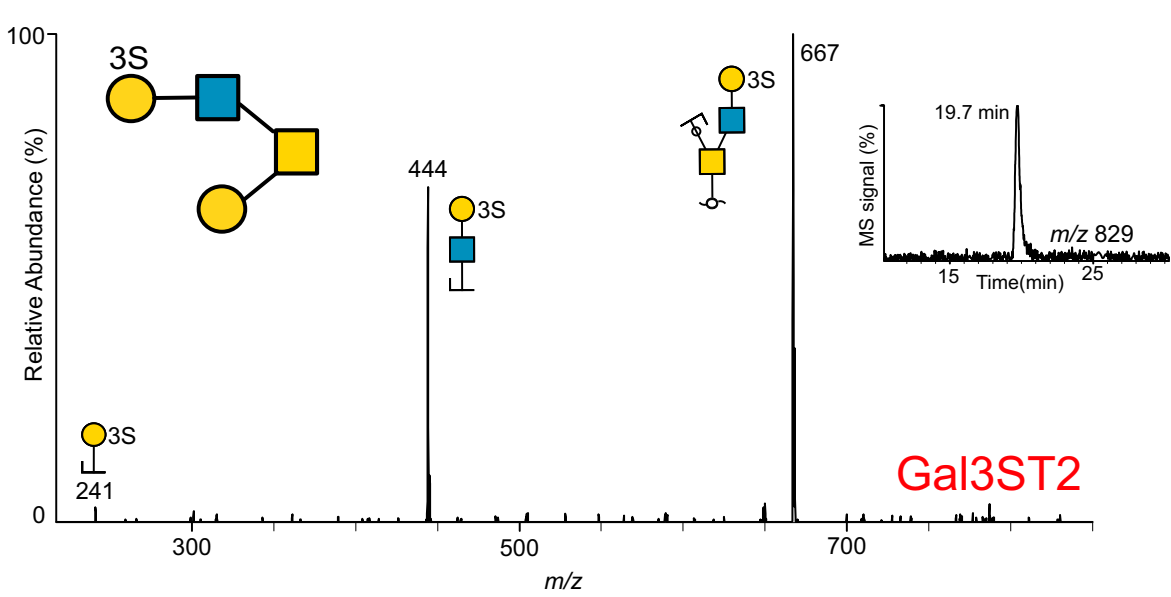

B

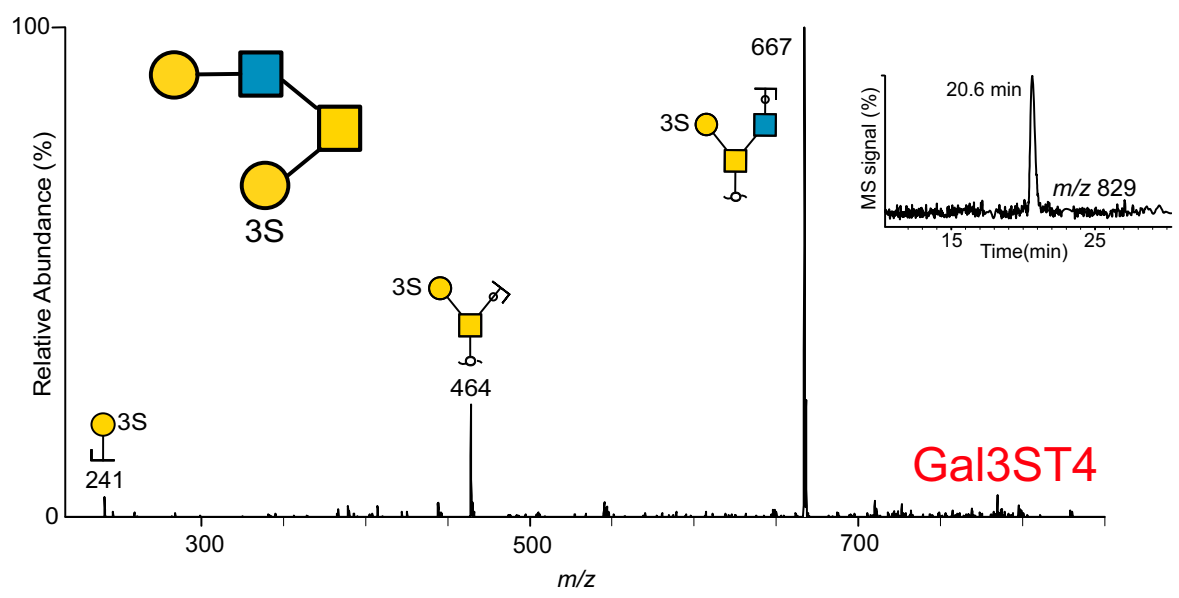

C
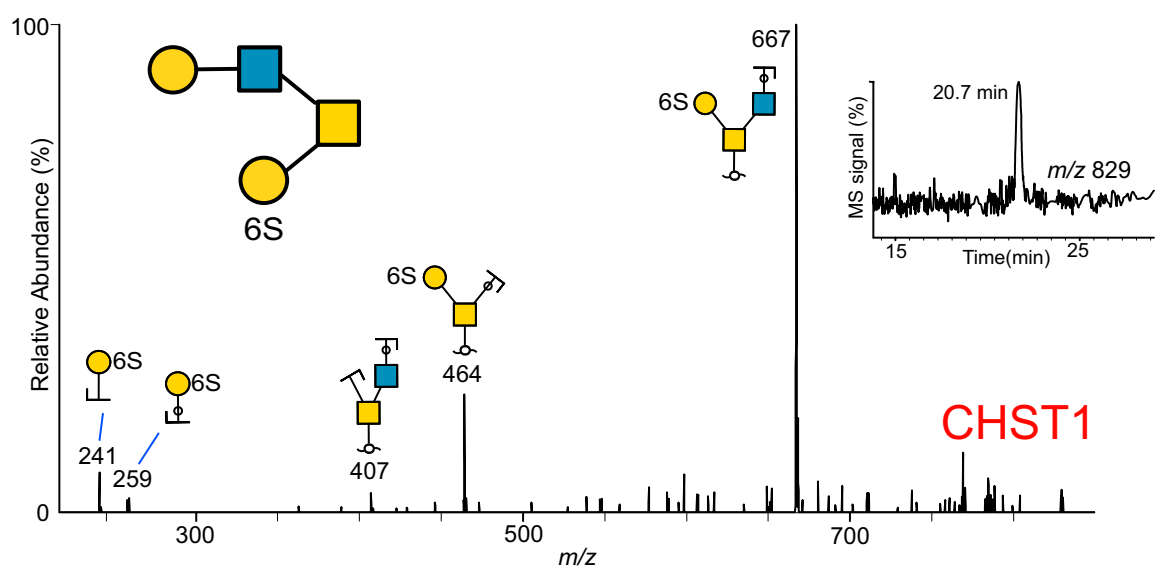

FIG. 7. $\mathrm{MS}^{2}$ spectra of sulfated core 2 O-glycans collected after fragmentation of the precursor ion at $\mathrm{m} / \mathrm{z} 829\left([\mathrm{M}-\mathrm{H}]^{-}\right)$from recombinantly produced PSGL-1/mlgG2b protein purified from CHO cells. PSGL-1 was cotransfected with core 2 transferase (GCNT1) and sulfotransferases Gal3ST2 $(A)$, Gal3ST4 $(B)$ or CHST1 $(C)$. For key to symbols, see Figure 1.

sulfate linked to a Hex residue, but the detected glycoforms were interpreted differently, as shown. The spectra of the glycoform produced by the Gal3ST2 sulfotransferase contained additional fragment ions at $\mathrm{m} / \mathrm{z} 444^{-}$, indicative of sulfate linked to Hex in the LacNAc unit on the $\mathrm{C} 6$ branch. The
$\mathrm{MS}^{2}$ spectra for the other two sulfotransferase glycoforms contained fragment ions at $\mathrm{m} / \mathrm{z} 464^{-}$, indicative of sulfate attached to Gal linked C-3 to the reducing sugar GalNAc. The two glycoforms generated by Gal3ST4 and CHST1, which differed only by C-3 or C-6 linked sulfate, had identical 
retention times and identical $\mathrm{MS}^{2}$ spectra. In addition to the 829 glycoforms, a few sialylated sulfated glycans were detected. The CHST1 sulfotransferase added two sulfates to a core 2 glycan detected at $\mathrm{m} / \mathrm{z} 599^{2-}$, the $\mathrm{MS}^{2}$ spectra was interpreted NeuAc $\alpha 2-3\left(\mathrm{HSO}_{3}-6\right) \mathrm{Gal} \beta 1-3\left(\mathrm{SO}_{3}-6 \mathrm{Gal} \beta 1-\right.$ 4 GlcNAc $\beta 1-6$ GalNAcol). This indicated that CHST1 could add sulfate to $\mathrm{Gal}$ also to the LacNAc C6-linked chain.

In summary, these experiments confirm that Gal3ST2, Gal3ST4, and CHST1 all add sulfate to Gal residues of the core 2 Gal $\beta 1-3($ Gal $\beta 1-3 G l c N A c \beta 1-6)$ GalNAc precursor present on the recombinantly produced PSGL $1 / \mathrm{mlg} 2 \mathrm{~b}$ in $\mathrm{CHO}$ cells, although with different fine specificities.

\section{Sulfotransferase Gal3ST2 Is Decreased in Tissue From Serous Ovarian Adenocarcinomas Compared With Serous Ovarian Adenomas}

To further investigate the pathological relevance of the three Gal-sulfotransferases in ovarian tissue, we screened patients ( $n=323$ ) using immunohistochemistry in TMA with specific antibodies targeting each of the three sulfotransferases. Representative immunohistochemical staining of tissue from two patients with malignant cancer and one benign patient is displayed in Figure $8 A$. The three graphs in Figure $8 B$ depict the groups consisting of selected patients with benign, borderline, and malignant epithelial ovarian tumors. The picture also includes a numerical score of a combined assessment of intensity of staining and \% positive cells (quick score QS). The results showed that all the three sulfotransferases are present in the three classes of ovarian tumors. No significant differences in the scoring were detected for CHST1 and Gal3ST4, whereas Gal3ST2 displayed a significant decrease $(p<0.0001)$ in expression comparing benign and borderline tissue with malignant tumor tissue (Fig. 8B). For malignant tumor, the expression of Gal3ST2 was even further attenuated in poorly differentiated tissue.

\section{DISCUSSION}

In our previous study, we concluded that fucosylated and sulfated O-glycans were potential biomarkers on ovarian serous cyst fluid proteins (15). However, the blood group associated enzymes FUT2 and FUT3 in secretions are not expressed in all individuals. In, for example, caucasians, approximately $80 \%$ express a functional FUT2, and approximately $90 \%$ express a functional FUT3. Blood group ABO fucosylation was found to be closely related to the mucinous subtype of OC. Here, we have focused on structural assignment of sulfated glycans from the serous type. The O-glycans were desialylated, and we performed data-dependent $\mathrm{MS}^{2}$, $\mathrm{MS}^{3}$, and $\mathrm{MS}^{4}$ experiments, which allowed structural elucidation. Desialylation was performed since sialic acid prevents detailed characterization of sulfation using MS in two ways. First, they give rise to more structural glycoforms, which lowers the relative abundance of each component. Second,
CID spectra of larger sialylated glycans are often not very informative, since most collision energy is spent on dissociation of the NeuAc glycosidic bonds.

In our previous study, we reported an overall estimated decrease in the abundance of sulfated glycans on serous cyst fluid proteins from patients diagnosed with malignant forms of OC (15). In the current study, we have performed a detailed structural assignment of sulfated $O$-glycans derived from cyst fluid proteins of two patients, one classified as benign, the second as malignant, grade "LGSC." The latter was chosen to include a malignant cancer, where we could detect significant amount of sulfation, while in the more aggressive "HGSC" tumor, their lower level of sulfation would have meant that sulfated glycans would be more difficult to characterize.

Our experiments revealed that ovarian glycoproteins carried sulfated core 1 and 20 -glycans consisting of up to ten residues, with 1 to 4 sulfates linked via hydroxyl groups to $\mathrm{N}$-acetylhexosamine residues or $\mathrm{C}-3$ and $\mathrm{C}-6$ to $\mathrm{Gal}$ in repeated LacNAc units on the C-6 branch. The presence of disulfated LacNAc units on glycoproteins from ovarian malignant tissue has previously been described by Shibata et al. (16). Based upon the LC-MS chromatograms of the desialylated sulfated oligosaccharides, no major qualitative or quantitative differences between the two samples could be observed.

Chromatographic profiles of short monosulfated glycans such as the core 1 sulfated T-antigens $\left(\mathrm{HSO}_{3}+\mathrm{Gal} \beta 1-3 \mathrm{Gal}-\right.$ $\mathrm{NAc}$ (Fig. 2) and sulfated core 2 glycans $\left(\mathrm{HSO}_{3}+\mathrm{Gal} \beta 1-3(\mathrm{Gal}-\right.$ GlcNAc $\beta 1-6)$ GalNAc) (Fig. 3) were similar between the two samples analyzed. This was also valid for the larger, sulfated glycans shown in Figure 5, with one exception. However, this exception could not be simply explained relating to an activity of a single sulfotransferase, and it remains as an unexplained observation that has to be addressed on a larger cohort of samples. However, as an overall conclusion, MS data indicate that it is the same bulk of sulfated glycans that are present in both the benign and malignant cyst fluid samples. Our previous results (15) that pointed toward a decreased level sulfation in malignant ovarian tumors suggest in this report to be mainly due to an overall decrease of sulfation rather than caused by altered activity of a single sulfotransferase, at least not a Gal-S transferase. However, in this report with the aim to thoroughly characterize the nature of sulfation in OC using only two patient samples, extended conclusion of the sulfoglycome in serous OC cannot be drawn.

At present, it is unclear to which proteins ovarian glycans are attached to and if the sulfation manifests differently on different protein cores. Prime protein candidates for sulfation are the mucin-type proteins revealed by immunohistochemistry and proteomics, such as CA125/MUC16, MUC5AC, MUC5B, MUC6, and MUC1 (3, 4).

We also investigated the relative abundance of sulfotransferases in ovarian tissue between different patient stages. There are 37 human Golgi membrane-bound sulfotransferases reported (24), many have primarily been studied 
A

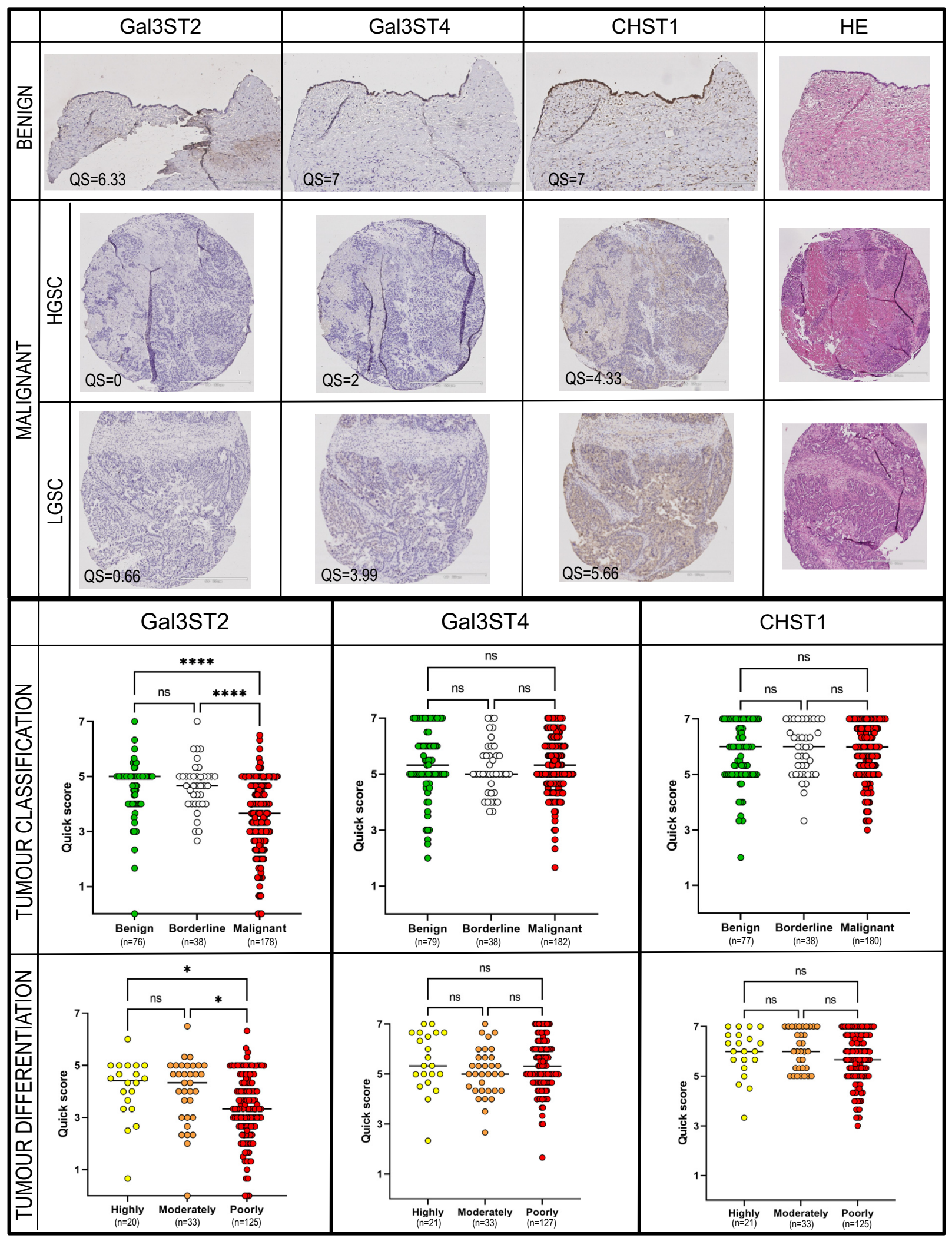

FIG. 8. Protein expression of sulfotransferases Gal3ST2, Gal3ST4, and CHST1 in benign and malignant serous ovarian tumor cells analyzed using immunohistochemistry and tissue micro arrays. $A$, representative antibody staining of tissue from patient diagnosed with serous cystadenoma, patient with low-grade serous carcinoma (LGSC, stage IIC, type 1) and patient with high-grade serous carcinoma (HGSC, 
due to their involvement in the sulfation of proteoglycans and/ or glycolipids, whereas less is known about their precursor substrate specificity and activity toward glycoproteins. Sulfated glycan motifs on glycoproteins are involved in interactions between leukocytes and the endothelium, such as $\mathrm{L}$-selectin mediated recirculation of lymphocytes to lymph nodes or leukocyte extravasation at sites of inflammation, and as ligands for sialic acid binding immunoglobulin-like receptors (Siglecs), which participate in the discrimination between "self" and "nonself" $(25,26)$. For mucins, which are commonly found as gel-forming or transmembrane proteins at mucosal surfaces, sulfated glycans have been proposed to be involved in the interaction and protection against microbiota $(27,28)$.

We detected sulfate linked to both $\mathrm{Gal}$ and GlcNAc in these samples and showed that sulfate could be linked both C-3 and C-6 to Gal. Since sulfation on Gal on glycoproteins is less well studied, we chose to explore three sulfotransferases: CHST1, Gal3ST2, and Gal3ST4. Extended mucin-type Oglycans are commonly composed of repeated poly $\mathrm{N}$-acetyllactosamine units [polyLacNAc; (-4GlcNAc $\beta 1-3 \mathrm{Gal} \beta 1-)_{n}$ ], which restricts Gal-3 sulfation only to terminally positioned residues, whereas Gal-6 sulfation occurs both on internal and terminal Gal. CHST1 (synonyms KSGal6ST, C6ST) was cloned 1997 (29). Its activity was confirmed and characterized by in vitro experiments observing the incorporation of ${ }^{35} \mathrm{~S}$ at the C6-position of Gal in keratan sulfate, but at a higher rate, to nonglycosaminoglycans on fetuin and to C-6 of Gal in sialyl $N$-acetyllactosamine oligosaccharides (NeuAc $\alpha 2-3 \mathrm{Gal} / \beta 1-$ $4 \mathrm{GlcNAc} \beta 1-)$ (30). It has been explored for its involvement in the biosynthesis of L-selectin ligands on high endothelial venules (31). During characterization of a monoclonal antibody HMOCC-1 against the ovarian clear cell cancer cell line RMG1, CHST1 was found to play a regulatory role in the formation of the sulfated glycan HMOCC-1 epitope (16). We showed here that CHST1 is extensively expressed in most OC tissues (Fig. 8). We confirmed its specificity acting on core 2 glycans on PSGL-1/lgG expressed in $\mathrm{CHO}$ cells, showing that the enzyme may add sulfate to $\mathrm{C}-6$ of both Gal residues of the core 2 tetrasaccharide NeuAc $\alpha 2-3 \mathrm{Gal} \beta 1-3(\mathrm{Gal} \beta 1-\mathrm{GlcNAc} \beta 1-6)$ GalNAc.

There are four Gal-3-sulfo transferases described of which three (Gal3ST2, Gal3ST3, and Gal3ST4) are found to act on glycoproteins, and all are found in ovarian tissue. Gal3ST2 (GP3ST) was shown to add sulfate to $\mathrm{C}-3$ of Gal in synthetic or purified oligosaccharides, preferentially on type 1 (-Gal $\beta 1$ 3GlcNAc-) and type 2 (-Gal $\beta 1-4 G \mid c N A c-)$ chains and to a lesser extent on the T-antigen (Gal $\beta 1-3 G a l N A c)$ (32). This is in line with our findings, where Gal3ST2 preferentially adds sulfate to the Gal in the LacNAc unit linked C-6 to GalNAc in the core 2 tetrasaccharide (Fig. 7). Analogous, we have previously reported core and branch-dependent specificity for $O$-linked glycans for the $\alpha 1,3$ fucosyltransferase family (33) using the $\mathrm{CHO}$-cell expression approach. Gal3ST4 was initially cloned and characterized 2001 by Seko et al. (34). He reported the incorporation of sulfate to C-3 of Gal linked to GalNAc in the core 2 saccharide Gal $\beta 1-3($ GlcNAc $\beta 1-6) G a l N A c$, which is concordant to our results. Gal3ST3 was not included in the current study, but is reported to act on core 2 glycans (35) and nextprot.org reports RNA levels present in ovarian tissue. The involvement of this enzyme in $\mathrm{OC}$ requires further investigation.

Our TMA study showed that protein expression levels of sulfotransferases Gal3ST4 and CHST1 remained the same in serous ovarian tissue from all tumor stages and degree of differentiation, whereas Gal3ST2 levels were decreased in malignant tissue. A decrease in sulfotransferase levels is in line with our previous findings where we used a mass spectrometric approach to semiquantify sulfated glycans from eight patients (15). However, our MS data indicates that there is no major change in the sulfated glycoforms in the samples from different patient groups, and it is likely that the overall sulfation decrease as previously indicated (15) must be related to more factors than Gal3ST2, which only can sulfate terminal Gal. There are additional sulfotransferase candidates, which may add sulfate to C-3 or C-6 in Gal of glycoproteins and are reported to be expressed in ovarian tissue (Gal3ST3 and CHST3). This may influence the overall sulfation, and further investigation of the role of sulfotransferases adding sulfate to C-6 of GlcNAc residues will provide complementary information about the role and regulation of sulfation in serous OC.

\section{DATA AVAILABILITY}

Raw MS data and Glycoworkbench files, which include fragment ion assignment for $\mathrm{OC}$ cyst fluid $\mathrm{O}$-glycan analyses, are available on glycopost (https://glycopost.glycosmos.org/) using the project ID: GPST000192. Structures with available MIRAGE information are available at Unicarb-DR (https:// unicarb-dr.glycosmos.org/references/453). Raw MS data for analyses of $O$-glycans from PSGL $1 / \mathrm{mlg} 2 \mathrm{~b}$ cotransfected with

stage IIIC, type 2). Immuno-positive staining is observed as brown coloring of tissue. Scale bars represent $200 \mu \mathrm{m}$ (benign tissue sections) or $500 \mu \mathrm{m}$ (malignant tissue sections). $B$, statistical evaluation of sulfotransferase expression after patient stratification. Patients were stratified based on their level of malignancy (benign ("BEN"), borderline, malignant ("MAL")). Malignant cases was also further subdivided based on level of differentiation ("highly," "moderately," or "poorly") according to WHO 2008 classification (37). Quick score (QS) scoring (0-7) for sulfotransferase expression was based upon manual evaluation and the sum of the proportion and intensity of staining (see Experimental Procedures section). Tissue micro array data was based upon the screening of tissue from 323 patients stratified based on malignancy level and level of differentiation. The clinicopathological data is compiled in supplemental Table S4. Tissue sections were analyzed in duplicates or triplicates. The bar graphs indicate the median value. For significance; ${ }^{* \star \star \star}$ means $p<0.001,{ }^{*}$ means $p<0.05$, ns means no significance. HE, hematoxylin-eosin. 
sulfotransferases Gal3T2, Gal3ST4, and CHST1 and with or without core 2 transferase GCNT1 are available at glycopost using the project IDS: GPST000198 and GPST000199, respectively. Structures with available MIRAGE information are available at Unicarb-DR, reference numbers 456 (core2+CHST1), 457 (core2+Gal3ST2), 458 (core2+Gal3ST4), 459 (core1+Gal3ST4), and 460 (core1+CHST1).

\section{Supplemental data-This article contains supplemental data.}

Funding and additional information-This study was funded by grants for (N. G. K.) from the Swedish state under the agreement between the Swedish government and the county council, the ALF-agreement (ALFGBG-722391, N. G. K., and ALFGBG-725381, K. S.), the Swedish Research Council (6212013-5895, N. G. K.), and Petrus and Augusta Hedlund's Foundation (M-2016-0353). V. V. was supported from Assar Gabrielsson's Foundation (FB16-102). The funding agencies were not involved in the conducted research.

Author contributions-C. M., J. L., C. J., J. H., K. S., and N. G. K. conceptualization; K. A. T., C. M., and K. S. data curation; K. A. T., C. M., J. L., and C. J. formal analysis; V. V., K. S., and N. G. K. funding acquisition; K. A. T., V. V., J. L., C. J., and B. W. investigation; V. V., J. L., C. J., J. H., and B. W. methodology; N. G. K. project management; C. M. and K. S. resources; K. S. and N. G. K. supervision; N. G. K. validation; K. A. T. and N. G. K. writingoriginal draft; K. S. and N. G. K. writing-review and editing.

Conflict of interest-The authors declare that they have no known competing financial interests or personal relationships that could have appeared to influence the work reported in this paper.

Abbreviations-The abbreviations used are: $\mathrm{CHO}$ cells, Chinese hamster ovary cells; Hex, hexose; HexNAc, $\mathrm{N}$-acetylhexosamine; HGSC, high-grade serous carcinoma; LC-MS, liquid chromatography-mass spectrometry; LGSC, low-grade serous carcinoma; OC, ovarian cancer; sLea, sialyl Lewis a; sLex, sialyl Lewis $\mathrm{x}$; T antigen, Gal $11-3 G$ alNAc-threonine/ serine; Tn antigen, GalNAc- $\alpha$-threonine/serine; TMA, tissue micro array.

Received July 5, 2021, and in revised from, August 19, 2021 Published, MCPRO Papers in Press, September 21, 2021, https://doi.org/ 10.1016/j.mcpro.2021.100150

\section{REFERENCES}

1. Lheureux, S., Gourley, C., Vergote, I., and Oza, A. M. (2019) Epithelial ovarian cancer. Lancet 393, 1240-1253

2. Wu, A. M. (2019) Glycan structures and their recognition roles in the human blood group ABH/li, Lea, b, x, y and Sialyl Lea,x active cyst glycoproteins. Glycoconj. J. 36, 495-507

3. Elzek, M. A., and Rodland, K. D. (2015) Proteomics of ovarian cancer: Functional insights and clinical applications. Cancer Metastasis Rev. 34, 83-96
4. Kristjansdottir, B., Levan, K., Partheen, K., Carlsohn, E., and Sundfeldt, K. (2013) Potential tumor biomarkers identified in ovarian cyst fluid by quantitative proteomic analysis, iTRAQ. Clin. Proteomics 10, 4

5. van Putten, J. P. M., and Strijbis, K. (2017) Transmembrane mucins: Signaling receptors at the intersection of inflammation and cancer. J. Innate Immun. 9, 281-299

6. Christiansen, M. N., Chik, J., Lee, L., Anugraham, M., Abrahams, J. L., and Packer, N. H. (2014) Cell surface protein glycosylation in cancer. Proteomics 14, 525-546

7. Duarte, H. O., Freitas, D., Gomes, C., Gomes, J., Magalhães, A., and Reis, C. A. (2016) Mucin-type O-glycosylation in gastric carcinogenesis. Biomolecules 6, 33

8. Jabbar, K. S., Arike, L., Verbeke, C. S., Sadik, R., and Hansson, G. C. (2018) Highly accurate identification of cystic precursor lesions of pancreatic cancer through targeted mass spectrometry: A phase Ilc diagnostic study. J. Clin. Oncol. 36, 367-375

9. Yin, B. W., and Lloyd, K. O. (2001) Molecular cloning of the CA125 ovarian cancer antigen: Identification as a new mucin, MUC16. J. Biol. Chem. 276, 27371-27375

10. Chen, K., Gentry-Maharaj, A., Burnell, M., Steentoft, C., Marcos-Silva, L., Mandel, U., Jacobs, I., Dawnay, A., Menon, U., and Blixt, O. (2013) Microarray glycoprofiling of CA125 improves differential diagnosis of ovarian cancer. J. Proteome Res. 12, 1408-1418

11. Ricardo, S., Marcos-Silva, L., Valente, C., Coelho, R., Gomes, R., and David, L. (2016) Mucins MUC16 and MUC1 are major carriers of SLe(a) and $\mathrm{SLe}(\mathrm{x})$ in borderline and malignant serous ovarian tumors. Virchows Arch. 468, 715-722

12. Ricardo, S., Marcos-Silva, L., Pereira, D., Pinto, R., Almeida, R., Soderberg, O., Mandel, U., Clausen, H., Felix, A., Lunet, N., and David, L. (2015) Detection of glyco-mucin profiles improves specificity of MUC16 and MUC1 biomarkers in ovarian serous tumours. Mol. Oncol. 9, 503-512

13. Vallen, M. J. E., van der Steen, S. C. H. A., van Tilborg, A. A. G., Massuger, L. F. A. G., and van Kuppevelt, T. H. (2014) Sulfated sugars in the extracellular matrix orchestrate ovarian cancer development: 'When sweet turns sour'. Gynecol. Oncol. 135, 371-381

14. Oliveira-Ferrer, L., Heßling, A., Trillsch, F., Mahner, S., and Milde-Langosch, K. (2015) Prognostic impact of chondroitin-4-sulfotransferase CHST11 in ovarian cancer. Tumour Biol. 36, 9023-9030

15. Vitiazeva, V., Kattla, J. J., Flowers, S. A., Linden, S. K., Premaratne, P., Weijdegard, B., Sundfeldt, K., and Karlsson, N. G. (2015) The O-linked glycome and blood group antigens $\mathrm{ABO}$ on mucin-type glycoproteins in mucinous and serous epithelial ovarian tumors. PLoS One 10, e0130197

16. Shibata, T. K., Matsumura, F., Wang, P., Yu, S., Chou, C. C., Khoo, K. H., Kitayama, K., Akama, T. O., Sugihara, K., Kanayama, N., Kojima-Aikawa, K., Seeberger, P. H., Fukuda, M., Suzuki, A., Aoki, D., et al. (2012) Identification of mono- and disulfated $\mathrm{N}$-acetyl-lactosaminyl oligosaccharide structures as epitopes specifically recognized by humanized monoclonal antibody HMOCC-1 raised against ovarian cancer. J. Biol. Chem. 287, 6592-6602

17. Schulz, B. L., Packer, N. H., and Karlsson, N. G. (2002) Small-scale analysis of O-linked oligosaccharides from glycoproteins and mucins separated by gel electrophoresis. Anal. Chem. 74, 6088-6097

18. Cherian, R. M., Jin, C., Liu, J., Karlsson, N. G., and Holgersson, J. (2015) A panel of recombinant mucins carrying a repertoire of sialylated O-glycans based on different core chains for studies of glycan binding proteins. Biomolecules 5, 1810-1831

19. Liu, J., Jin, C., Cherian, R. M., Karlsson, N. G., and Holgersson, J. (2015) Oglycan repertoires on a mucin-type reporter protein expressed in $\mathrm{CHO}$ cell pools transiently transfected with O-glycan core enzyme cDNAs. J. Biotechnol. 199, 77-89

20. Ceroni, A., Maass, K., Geyer, H., Geyer, R., Dell, A., and Haslam, S. M. (2008) GlycoWorkbench: A tool for the computer-assisted annotation of mass spectra of glycans. J. Proteome Res. 7, 1650-1659

21. Barone, A., Linder, A., Mateoiu, C., Köster Larsen, R., Blixt, O., Teneberg, S., and Sundfeldt, K. (2020) Evaluation of sialyl-lactotetra as a marker for epithelial ovarian tumors. Front. Oncol. 10, 561888

22. Thomsson, K. A., Karlsson, H., and Hansson, G. C. (2000) Sequencing of sulfated oligosaccharides from mucins by liquid chromatography and electrospray ionization tandem mass spectrometry. Anal. Chem. 72, 4543-4549 
23. Flowers, S. A., Ali, L., Lane, C. S., Olin, M., and Karlsson, N. G. (2013) Selected reaction monitoring to differentiate and relatively quantitate isomers of sulfated and unsulfated core $1 \mathrm{O}$-glycans from salivary MUC7 protein in rheumatoid arthritis. Mol. Cell. Proteomics 12, 921-931

24. Langford, R., Hurrion, E., and Dawson, P. A. (2017) Genetics and pathophysiology of mammalian sulfate biology. J. Genet. Genomics 44, 7-20

25. Uchimura, K., and Rosen, S. D. (2006) Sulfated L-selectin ligands as a therapeutic target in chronic inflammation. Trends Immunol. 27, 559-565

26. Macauley, M. S., Crocker, P. R., and Paulson, J. C. (2014) Siglec-mediated regulation of immune cell function in disease. Nat. Rev. Immunol. 14, 653-666

27. Al-Saedi, F., Vaz, D. P., Stones, D. H., and Krachler, A. M. (2017) 3Sulfogalactosyl-dependent adhesion of Escherichia coli HS multivalent adhesion molecule is attenuated by sulfatase activity. J. Biol. Chem. 292, 19792-19803

28. Benjdia, A., Martens, E. C., Gordon, J. I., and Berteau, O. (2011) Sulfatases and a radical S-adenosyl-L-methionine (AdoMet) enzyme are key for mucosal foraging and fitness of the prominent human gut symbiont, Bacteroides thetaiotaomicron. J. Biol. Chem. 286, 25973-25982

29. Fukuta, M., Inazawa, J., Torii, T., Tsuzuki, K., Shimada, E., and Habuchi, O. (1997) Molecular cloning and characterization of human keratan sulfate Gal-6-sulfotransferase. J. Biol. Chem. 272, 32321-32328

30. Torii, T., Fukuta, M., and Habuchi, O. (2000) Sulfation of sialyl N-acetyllactosamine oligosaccharides and fetuin oligosaccharides by keratan sulfate Gal-6-sulfotransferase. Glycobiology 10, 203-211

31. Bistrup, A., Bhakta, S., Lee, J. K., Belov, Y. Y., Gunn, M. D., Zuo, F. R., Huang, C. C., Kannagi, R., Rosen, S. D., and Hemmerich, S. (1999)
Sulfotransferases of two specificities function in the reconstitution of high endothelial cell ligands for L-selectin. J. Cell Biol. 145, 899-910

32. Honke, K., Tsuda, M., Koyota, S., Wada, Y., lida-Tanaka, N., Ishizuka, I., Nakayama, J., and Taniguchi, N. (2001) Molecular cloning and characterization of a human beta-Gal-3'-sulfotransferase that acts on both type 1 and type 2 (Gal beta 1-3/1-4GlcNAc-R) oligosaccharides. J. Biol. Chem. 276, 267-274

33. Löfling, J., and Holgersson, J. (2009) Core saccharide dependence of sialyl Lewis X biosynthesis. Glycoconj. J. 26, 33-40

34. Seko, A., Hara-Kuge, S., and Yamashita, K. (2001) Molecular cloning and characterization of a novel human galactose 3-O-sulfotransferase that transfers sulfate to gal beta 1->3galNAc residue in O-glycans. J. Biol. Chem. 276, 25697-25704

35. El-Fasakhany, F. M., Uchimura, K., Kannagi, R., and Muramatsu, T. (2001) A novel human gal-3-O-sulfotransferase: Molecular cloning, characterization, and its implications in biosynthesis of (SO(4)-3)Galbeta14(Fucalpha1-3)GlcNAc. J. Biol. Chem. 276, 26988-26994

36. Varki, A., Cummings, R. D., Aebi, M., Packer, N. H., Seeberger, P. H., Esko, J. D., Stanley, P., Hart, G., Darvill, A., Kinoshita, T., Prestegard, J. J., Schnaar, R. L., Freeze, H. H., Marth, J. D., Bertozzi, C. R., et al. (2015) Symbol nomenclature for graphical representations of glycans. Glycobiology 25, 1323-1324

37. Swerdlow, S. H., Campo, E., Harris, N. L., Jaffe, E. S., Pileri, S. A., Stein, H., Thiele, J., Vardiman, J. W., eds. (2008) WHO classification of tumours of haematopoietic and lymphoid tissues. WHO Press, Geneva, Switzerland 